\title{
TCF21 and AP-1 interact through epigenetic modifications to regulate coronary artery disease gene expression
}

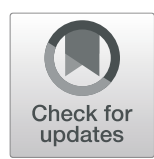

Quanyi Zhao ${ }^{1}$, Robert Wirka ${ }^{1}$, Trieu Nguyen ${ }^{1}$, Manabu Nagao ${ }^{1}$, Paul Cheng ${ }^{1}$, Clint L. Miller ${ }^{2,3,4}$, Juyong Brian Kim', Milos Pjanic ${ }^{1}$ and Thomas Quertermous ${ }^{1 *}$

\begin{abstract}
Background: Genome-wide association studies have identified over 160 loci that are associated with coronary artery disease. As with other complex human diseases, risk in coronary disease loci is determined primarily by altered expression of the causal gene, due to variation in binding of transcription factors and chromatin-modifying proteins that directly regulate the transcriptional apparatus. We have previously identified a coronary disease network downstream of the disease-associated transcription factor TCF21, and in work reported here extends these studies to investigate the mechanisms by which it interacts with the AP-1 transcription complex to regulate local epigenetic effects in these downstream coronary disease loci.

Methods: Genomic studies, including chromatin immunoprecipitation sequencing, RNA sequencing, and proteinprotein interaction studies, were performed in human coronary artery smooth muscle cells.

Results: We show here that TCF21 and JUN regulate expression of two presumptive causal coronary disease genes, SMAD3 and CDKN2B-AS1, in part by interactions with histone deacetylases and acetyltransferases. Genome-wide TCF21 and JUN binding is jointly localized and particularly enriched in coronary disease loci where they broadly modulate H3K27Ac and chromatin state changes linked to disease-related processes in vascular cells. Heterozygosity at coronary disease causal variation, or genome editing of these variants, is associated with decreased binding of both JUN and TCF21 and loss of expression in cis, supporting a transcriptional mechanism for disease risk.

Conclusions: These data show that the known chromatin remodeling and pioneer functions of AP-1 are a pervasive aspect of epigenetic control of transcription, and thus, the risk in coronary disease-associated loci, and that interaction of AP-1 with TCF21 to control epigenetic features, contributes to the genetic risk in loci where they co-localize.
\end{abstract}

Keywords: Transcription, Epigenomics, TCF21, AP-1, Histone acetyltransferase, Deacetylase

\section{Background}

Collaborative efforts from multiple large genome-wide association studies (GWAS) in the past decade have identified 161 independent genomic loci significantly associated with CAD in humans [1-4]. While a small subset of coronary artery disease (CAD) variants alter specific protein composition, the majority of the identified lead SNPs (single nucleotide polymorphisms) are

\footnotetext{
* Correspondence: tomq1@stanford.edu

${ }^{1}$ Division of Cardiovascular Medicine and Cardiovascular Institute, School of Medicine, Stanford University, 300 Pasteur Dr., Falk CVRC, Stanford, CA 94305, USA

Full list of author information is available at the end of the article
}

located in non-coding enhancer regions, suggesting that variation in cellular transcriptional response rather than protein function underlies the majority of heritable disease risk [5]. Although identified genetic loci offer great promise in advancing our understanding of CAD pathophysiology, these transcriptional regulatory regions are poorly annotated, and thus, the mechanisms by which the causal variants affect causal gene expression are poorly understood. Identifying the transcriptional mechanisms shared among multiple CAD risk loci will not only provide new insights into the pathophysiology of CAD, but will also allow us to identify core mechanisms for intervention to modify disease risk.

(C) The Author(s). 2019 Open Access This article is distributed under the terms of the Creative Commons Attribution 4.0 International License (http://creativecommons.org/licenses/by/4.0/), which permits unrestricted use, distribution, and 
TCF21 is a basic helix-loop transcription factor critical in cardiovascular development and disease [2-4, 6-14]. In the context of atherosclerosis, an important feature of TCF21 biology is the fact that it regulates fundamental cellular differentiation events in the developing epicardium, serving as a determining factor for the divergence between coronary vascular smooth muscle cell (SMC) and cardiac fibroblast lineages $[15,16]$. Embryonic loss of Tcf 21 has been linked to premature differentiation of SMC from the pericardium, resulting in decreased migration into the myocardium [16]. TCF21 has been extensively associated with CAD risk, having been associated with disease in multiple racial ethnic groups [14, $17,18]$. Vascular smooth muscle cell expression quantitative trait locus (eQTL) studies [19], as well mechanistic follow-up studies, have identified TCF21 as the causal gene in the CAD-associated locus at 6q23.2 [20] and suggested that expression of this gene is protective toward CAD risk [21]. Further, TCF21 has been shown to be expressed in cells that migrate into the developing plaque and contribute to the protective fibrous cap [22]. TCF21 downstream target regions are enriched for known CAD risk loci, suggesting that TCF21 plays a central role in regulating risk in other loci to effect the biology of atherosclerotic plaques [23].

The activated protein-1 (AP-1) family of proteins includes a number of bZip transcription factors that bind primarily as heterodimers to a well-characterized canonical DNA sequence. Expression of these early response genes (e.g., FOS, FOSB, JUN, JUND) is initiated in all cell types when growth factors activate their cognate receptor tyrosine kinases, inducing the Ras/MAPK intracellular kinase cascade, promoting a variety of cell state changes [24-26]. These AP-1 site-binding proteins have been shown to play a central role in specific lineage-restricted enhancer selection and function [27, 28]. FOS/JUN factors select enhancers with cell type-specific transcription factors (TFs) by collaboratively binding to histone bound enhancers and recruiting the BAF chromatin remodeling complex to establish accessible chromatin [29]. At a more general level, AP-1 factors have been shown to disrupt the nucleosomal structure and regulate chromatin accessibility in various cell types [29-31]. In the context of complex diseases, variation in AP-1 binding sites has been shown to mediate genetic risk [20, 32-35]. An AP-1 site in an intron of the SMAD3 gene has been implicated in the association of this gene with CAD, as well as an autoimmune disease [20,33-35]. Important for this discussion, AP-1 binding sites in two different CAD-associated alleles that regulate expression of TCF21 have been shown to alter risk in Caucasian and East Asian populations $[14,17,20]$.

In studies reported here, we have investigated the interaction of transcription factors TCF 21 and AP-1 at loci identified by GWAS to regulate risk for the CAD phenotype, focusing on $15 \mathrm{q} 22.33$ and $9 \mathrm{p} 21.3$. Studies employing eQTL mapping, allele-specific expression, co-localization of eQTL and GWAS, in vitro experiments, cell culture, and animal models have identified SMAD3 [19, 33, 36, 37] and CDKN2B-AS1 (CDKN2BAS) [38-42] as putative causal genes in these loci. We show that TCF21 and AP-1 regulate expression of these genes by regulating chromatin accessibility through interactions with histone acetyltransferases and histone deacetylases and also through protein-protein interactions. We subsequently generalize our finding on these two loci genome-wide and demonstrate that interaction between AP1 and TCF21 controls epigenetic features in regions where they co-localize. These regions are enriched for CAD genetic risk loci, suggesting a critical role for this interaction in modifying risk for CAD.

\section{Methods}

\section{Primary cell culture and reagents}

Primary human coronary artery smooth muscle cells (HCASMCs) derived from normal human donor hearts were purchased from three different manufacturers Lonza, PromoCell, and Cell Applications (all tested negative for mycoplasma contamination) at passage 2 and were maintained in growth-supplemented smooth muscle basal media (Lonza \# CC-3182) according to the manufacturer's instructions. All experiments were performed on HCASMCs between passages 4 and 7. Antibodies used for ChIPseq and ChIP-quantitative PCR (qPCR) were all pre-validated according to ChIPseq guidelines and ENCODE best practices. Purified mouse monoclonal antibodies against human JUN (sc-74543) and p300 (sc-48343) were purchased from Santa Cruz. Purified rabbit polyclonal antibody against H3K27ac (ab4729), HDAC1 (ab7028), and HDAC2 (ab7029) were purchased from Abcam. Purified rabbit polyclonal antibody against human TCF21 (HPA013189) was purchased from Sigma.

\section{Knockdown and overexpression}

JUN (s7659) and p300 (s534247) silencer select siRNAs were purchased from Thermo Fisher Scientific. TCF21 (SR321985) Trilencer-27 siRNAs were purchased from OriGene. siRNA transfection was performed using Lipofectamine RNAiMAX (Life Technologies). For each well treated with the siRNAs or scrambled control (Life Technologies, \#4390843), the final concentration was $20 \mathrm{nM}$. HCASMCs were seeded in 6-well plates and grown to $50 \%$ confluence before siRNA transfection. HCASMCs were transfected with the siRNA or scrambled control for 6-8 $\mathrm{h}$ and subsequently collected and processed for RNA isolation after $48 \mathrm{~h}$ of transfection using the RNeasy mini kit (Qiagen 74106). 
For lentivirus transduction study, control (titer $5.68 \times 10^{7}$ ), TCF21 cDNA (titer $3.84 \times 10^{6}$ ), and shRNA (titer $2.59 \times 10^{7}$ ) virus were used as described before [22]. JUN shRNA (titer $8.4 \times 10^{8}$ ) virus was purchased from OriGene (TL320397). HCASMCs were transduced with the virus at a density of $5 \times 10^{5}$ cells per T75-flask. Cells were changed to medium with supplements $12 \mathrm{~h}$ after transduction and cultured for an additional $48 \mathrm{~h}$. The transduction efficiency was assessed by quantifying the percentage of GFP-positive cells. Transduction efficiencies were more than $90 \%$.

\section{RNA isolation and qRT-PCR}

RNA for all samples was extracted using the RNeasy mini kit (Qiagen 74106). HCASMC RNA (500 ng) was reverse transcribed using the High-Capacity RNA-to-cDNA Synthesis kit (Applied Biosystems 4387406). Quantitative PCR of the cDNA samples was performed on a ViiA7 Real-Time PCR system, and gene expression levels were measured using SYBR green assays (Applied Biosystems 4368706) using custom-designed primers and normalized to $A C T B$ levels. Comparison between two groups was performed using the Student's $t$ test, and comparisons between the three groups were performed using ANOVA.

qPCR primers were designed as follows: $S M A D 3$, Fwd: CCATCTCCTACTACGAGCTGAA, Rev: CACT GCTGCATTCCTGTTGAC, Amplicon: $149 \mathrm{bp}$; CDKN2BAS, Fwd: CTATCCGCCAATCAGGAGGC, Rev: GCGTGCAGC GGTTTAGTTTA, Amplicon: 103 bp; CDKN2B, Fwd: GGGACTAGTGGAGAAGGTGC, Rev: CATCATCAT GACCTGGATCGC, Amplicon: $97 \mathrm{bp;} J U N$, Fwd: TCCA AGTGCCGAAAAAGGAAG, Rev: CAGCACAATGAAG ATCAAGA, Amplicon: 78 bp; TCF21, Fwd: TCCT GGCTAACGACAAATACGA, Rev: TTTCCCGGCCACCA TAAAGG, Amplicon: 77 bp; EP300, Fwd: GCTTCAGAC AAGTCTTGGCAT, Rev: ACTACCAGATCGCAGCAAT TC, Amplicon: $79 \mathrm{bp}$.

\section{ChIP assay}

Briefly, approximately 4e6 HCASMC cells were fixed with $1 \%$ formaldehyde and quenched by glycine. The cells were washed three times with PBS and then harvested in ChIP lysis buffer (50 mM Tris- $\mathrm{HCl}, \mathrm{pH} 8,5 \mathrm{mM}$ EDTA, 0.5\% SDS). Crosslinked chromatin was sheared for $3 \times 1 \mathrm{~min}$ by sonication (Branson SFX250 Sonifier) before extensive centrifugation. Four volumes of ChIP dilution buffer (20 mM Tris- $\mathrm{HCl}, \mathrm{pH} 8.0,150 \mathrm{mM} \mathrm{NaCl}, 2 \mathrm{mM}$ EDTA, $1 \%$ Triton $\mathrm{X}-100$ ) was added to the supernatant. The resulting lysate was then incubated with Dynabeads ${ }^{\text {Tx }}$ Protein G (Thermo Scientific, 10009D) and antibodies at $4{ }^{\circ} \mathrm{C}$ overnight. Beads were washed once with buffer 1 (20 mM Tris pH 8, 2 mM EDTA, $150 \mathrm{mM} \mathrm{NaCl}, 1 \%$ Triton X100, 0.1\% SDS), once with buffer 2 (10 mM Tris pH $8,1 \mathrm{mM}$ EDTA, $500 \mathrm{mM} \mathrm{NaCl}, 1 \%$ Triton
X100, 0.1\% SDS), once with buffer 3 (10 mM Tris pH 8, $1 \mathrm{mM}$ EDTA, $250 \mathrm{mM} \mathrm{LiCl}$ 1\% NP40, 1\% sodium deoxycholate monohydrate), and twice with TE buffer. DNA was eluted by ChIP elution buffer (0.1 M NaHCO 3 , $1 \%$ SDS, $20 \mu \mathrm{g} / \mathrm{ml}$ proteinase $\mathrm{K})$. The elution was incubated at $65^{\circ} \mathrm{C}$ overnight, and DNA was extracted with a DNA purification kit (Zymo D4013). The purified DNA was assayed by quantitative PCR with ABI ViiA 7 and Power SYBR Green Master Mix (ABI 4368706). For serial ChIP, the DNA-protein complex of the first IP was eluted with elution buffer containing no proteinase $\mathrm{K}$ after washing. The elution was diluted $1: 9$ by dilution buffer followed by the second IP and washing steps. Heterozygous genotypes at the candidate loci were determined using TaqMan SNP genotyping qPCR assays (Thermo Fisher Scientific C_33991343_10). Assays were repeated at least three times. Data shown were average values \pm SD of representative experiments.

qPCR primers were designed as follows: SMAD3-1, Fwd: GGTTGACCCGTTGCATGTTA, Rev: GGAGAGGTG AAGAGGGCAAA, Amplicon: $129 \mathrm{bp} ; S M A D 3-2$, Fwd: AGAGGGCAGAGAGAGGATAC, Rev: AGTCTTATC TGCCGGCAAAC, Amplicon: 142 bp; CDKN2BAS, Fwd: GCATTGAGAAGTCCAGCCAG, Rev: GCAGCAACT TCGAAGCTTGA, Amplicon: 136 bp.

\section{Immunoprecipitation and western blotting}

Nuclear complex Co-IP kit (Active Motif 54001) was used for co-immunoprecipitation following the manufacturer instructions. ChIP samples were eluted with $1 \times$ Laemmli buffer (Bio-Rad 161-0747) containing Halt protease inhibitor cocktail (Thermo Fisher Scientific 78429) instead of elution buffer for western blotting. The elution was incubated at $65^{\circ} \mathrm{C}$ for $6 \mathrm{~h}$ and then boiled at $95^{\circ} \mathrm{C}$ for $2 \mathrm{~min}$. Whole cell lysate samples were harvested at $4{ }^{\circ} \mathrm{C}$ using $1 \times$ Laemmli buffer and boiled at $95^{\circ} \mathrm{C}$ for $10 \mathrm{~min}$.

The 5-40- $\mu$ l sample was loaded onto a $4-15 \%$ gradient SDS-PAGE gel (Bio-Rad 4561084DC). Samples were transferred to polyvinylidene difluoride membrane (Thermo Fisher Scientific LC2002) for $1 \mathrm{~h}$ at $300 \mathrm{~mA}$ at $4{ }^{\circ} \mathrm{C}$ and blocked with $5 \%$ milk in Tris-buffered saline and $0.1 \%$ Tween 20 (TBST, Bio-Rad 1706435) at room temperature. The membranes were hybridized with the following primary antibodies: mouse Myc-Tag antibody (CST 2276S), rabbit SMAD3 antibody (CST 9523S), rabbit c-JUN antibody (CST 9165S), p300, HDAC1, and HDAC2 antibodies as described above. Rabbit GAPDH antibody (Sigma G9545-200UL) was used as the loading control. Anti-mouse HRP (Jackson ImmunoResearch 115-035-008, 115-035-003, and 115-035-174) or antirabbit HRP (Jackson ImmunoResearch 211-032-171, 111-035-008, and 111-035-003) secondary antibodies were used at a concentration of 1:10000 and diluted in 
$5 \%$ milk containing $0.1 \%$ Tween 20 . Bands were detected using SignalBoost ${ }^{\mathrm{TM}} I m m u n o r e a c t i o n$ Enhancer Kit (Millipore 407207) per manufacturer's instructions on the LI-COR Odyssey imaging system.

\section{ChIPseq analysis}

ChIP was performed with approximately 4xE6 HCAMSC cells using H3K27ac antibody (Abcam ab4729) under JUN siRNA or TCF21 shRNA knockdown described in the "Knockdown and overexpression" section. Two biological replicate DNA were combined for library preparation. Libraries were prepared with KAPA Hyper Prep kit (KK8502). ChIPseq libraries were sequenced on HiSeq X10 for $150 \mathrm{bp}$ paired-end sequencing. Quality control of ChIPseq data was performed using Fastqc, and then low-quality bases and adaptor contamination were trimmed by cutadapt. Filtered reads were mapped to hg19 using BWA mem algorithm. Duplicate reads were marked by Picard Markduplicate module and removed with unmapped reads by samtools view $-f 2-F$ 1804. macs 2 callpeak was used for peaks calling with --broad parameters and input as control. macs 2 bdgdiff was used for differential peaks calling with default parameters. The fastq files of TCF21 (SRR1573744\&SRR1573745), JUN (SRR1573749), and HNF1A (SRR5339317) ChIPseq were extracted from GEO database by fastq-dump. Similar methods (except --broad parameter in macs2) were used in quality control, alignment, filtering, and peak calling. The average fragment size for the JUN ChIPseq was $186 \mathrm{bp}$, and for TCF21 was $196 \mathrm{bp}$.

\section{ATACseq analysis}

Approximately $5 \mathrm{xE} 4$ fresh HCAMSC cells, each with TCF21 shRNA knockdown or lentivirus overexpression, and JUN shRNA knockdown (described in the "Knockdown and overexpression" section) were collected by centrifugation at $500 \mathrm{~g}$ and washed twice with cold PBS. Nuclei-enriched fractions were extracted with cold resuspension buffer $(0.1 \% \mathrm{NP}-40,0.1 \%$ Tween 20 , and $0.01 \%$ Digitonin) and washed out with $1 \mathrm{ml}$ of cold resuspension buffer containing $0.1 \%$ Tween 20 only. Nuclei pellets were collected by centrifugation and resuspended with transposition reaction buffer containing Tn5 transposases (Illumina Nextera). Transposition reactions were incubated at $37^{\circ} \mathrm{C}$ for $30 \mathrm{~min}$, followed by DNA purification using the DNA Clean-up and Concentration kit (Zymo D4013). Libraries were amplified using Nextera barcodes and high-fidelity polymerase (NEB M0541S) and purified using Agencourt Ampure XP beads (Beckman Coulter A63880) double-size selection (0.5X:0.9X). For qPCR experiments, the purified DNA was quantified with ABI ViiA 7 and Power SYBR Green Master Mix (ABI 4368706) and normalized by genomic DNA which extracted using Quick-DNA Microprep Kit
(Zymo D3020). Assays were repeated at least three times. Data shown were average values \pm SD of representative experiments.

Libraries were sequenced on HiSeq X10 for 150-bp paired-end sequencing. Raw fastq files were evaluated with fastqc, and then low-quality bases and adaptor contamination were trimmed by cutadapt. Reads were mapped to hg19 using bowtie2. Duplicate reads were marked by Picard Markduplicate module and removed with unmapped or mitochondrial reads by samtools. bedtools was used to generate BED file from filtered reads followed by Tn 5 shifting with awk. macs 2 callpeak with --broad and SICER-rb.sh with "W200 G600 E1000" parameters were used for peak calling. macs 2 bdgdiff and SICER-df-rb.sh with FDR cutoff 0.05 were used for differential peak comparison in JUN or TCF21 disrupted samples. Bigwig files were generated for University of California Santa Cruz (UCSC) Genome Browser visualization.

\section{Peak co-localization and enrichment analysis}

The intersectBed was used to find at least $1 \mathrm{bp}$ overlapped peaks between H3K27ac, ATAC, TCF21, and JUN. We combined the open chromatin regions from ENCODE data as intersection background. $P$ values were calculated by Fisher's exact test. For the common (conserved) open chromatin of ENCODE, we defined the peaks which have overlaps in more than $50 \%$ of the cell lines (70 out of 126 lines) that are conserved. These peaks in each line were merged to get the final common open chromatin regions. For enrichment level analysis, reads of control/treatment samples which mapped on peaks were counted by intersectBed and normalized by per million (RPM). JUN, TCF21 peaks, or open chromatin regions were clustered using the hierarchical algorithm, and reads centering on these peaks $( \pm 2 \mathrm{~kb})$ were plotted with deeptools.

\section{Cis-regulatory functional enrichment and network analysis}

We utilized the Genomic Regions Enrichment of Annotations Tool (GREAT 3.0) to analyze the detected peaks, with the parameter "single nearest gene," which is within $50 \mathrm{~kb}$ to nearest genes. Gene ontology from GREAT output was analyzed by DAVID. KEGG pathways, biological processes, and GAD disease enrichment analysis was carried out using default settings. The HOMER findMotifsGenome.pl script was employed to search for known TRANSFAC motifs and to generate de novo motifs. PWMScore was used for position weight matrix scan. We obtained full-length JUN (MA0488.1) and TCF21 (MA0832.1) motifs from JASPAR. 
Association of CAD loci and GWAS enrichment analysis HCAMSC eQTL data came from a genome-wide association of gene expression with imputed common variation identified in $52 \mathrm{HCASMC}$ lines studied with whole genome RNA sequencing and 30x whole-genome sequencing [19]. CARDIoGRAMplusC4D variant data was from 1000 Genome-based GWAS meta-analyses [43]. Coronary artery (CA) tissue-specific SNP gene association data was obtained from GTEx V6p. We intersected open chromatin region which regulated by TCF21 and AP-1 with these three datasets; the significance cutoff for GTEx is nominal cis-eQTL $P<0.05, F D R<0.05$ for HCAMSC eQTL, and beta $P<0.05$ for CARDIoGRAMplusC4D. Overlap of TCF21-AP1 co-regulated open chromatin region and GWAS Catalog SNPs was performed with bed2GwasCatalogBinomialMod1Ggplot script from gwasanalytics package. The calculation criteria of this script were described previously [44].

\section{Statistical analysis}

All experiments were performed by the investigators blinded to the treatments/conditions during the data collection and analysis, using at least two independent biological replicates and treatments/conditions in technical triplicate. $R$ or GraphPad Prism was used for statistical analysis. For motifs and gene enrichment analyses, we used the cumulative binomial distribution test. For overlapping of genomic regions or gene sets, we used Fisher's exact test. For comparisons between two groups of equal sample size (and assuming equal variance), an unpaired two-tailed Student's $t$ test was performed or in cases of unequal sample sizes or variance, Welch's unequal variances $t$ test was performed, as indicated. $P$ values $<0.05$ were considered statistically significant. For multiple comparison testing, one-way analysis of variance (ANOVA) accompanied by Tukey's post hoc test were used as appropriate. All error bars represent standard error of the mean (SE). Number of stars for the $P$ values in the graphs: ***** $P<0.0001$, *** $P<0.001$, * $P<0.01$, ${ }^{*} P<0.05$.

\section{Generation and analysis of CRISPR lines}

HEK293 cell line was used for genome editing. Cells were maintained in DMEM containing 10\% FBS prior to and during transfection. Cells were seeded into 6-well plates at $2 \times 10^{5}$ cells/well and were transfected with $2 \mu \mathrm{g}$ sgRNA/Cas9-GFP using $7.5 \mu$ l Lipofectamine 3000 and $4 \mu \mathrm{l}$ P3000 reagent per well $24 \mathrm{~h}$ after seeding. Two days after transfection cells were sorted using a Digital Vantage (BD Biosciences). GFP-positive cells were singly sorted into two 96-well plates and allowed to grow until clones reached about $70 \%$ confluence, about 2 weeks. All wells with colonies were marked and then split 1:2 into two fresh 96-well plates. Once a majority of the wells obtained $80 \%$ confluence, one plate of cells was frozen, and the other was used for genomic DNA extraction using Qiagen DNeasy Blood and Tissue Kit as per manufacturer's instructions. The targeted region was amplified using $10 \mu \mathrm{l}$ of gDNA, specific primers SMAD3_650F, and ChipSeqR and Taq DNA Polymerase (NEB) as per manufacturer's instructions. Cycling conditions were as follows: $94{ }^{\circ} \mathrm{C}$ for $3 \mathrm{~min}, 35$ cycles of $94{ }^{\circ} \mathrm{C}$ for $1 \mathrm{~min}, 63{ }^{\circ} \mathrm{C}$ for $30 \mathrm{~s}, 72{ }^{\circ} \mathrm{C}$ for $45 \mathrm{~s}$, and one cycle of $72{ }^{\circ} \mathrm{C}$ for $5 \mathrm{~min}$. All wells showing a positive band at about 500 bp were Sanger sequenced with SMAD3_650F primer, and the sequence was analyzed for indels. The methods of RNA extraction and expression analysis were described above.

\section{RNA seq analysis}

HCASMC were transfected with TCF21 or scrambled siRNA using the method described in the "Knockdown and overexpression" for three biological replicates. The cells were then collected $48 \mathrm{~h}$ after transfection and processed using RNeasy kit (Qiagen 74106) for RNA isolation. The cells were sent to Novogene for sample QC, library preparation, and sequencing. All samples passed QC, and 250-300 bp insert cDNA libraries were prepared for each sample. Subsequently, sequencing was performed on a Novaseq 6000 platform with paired-end $150 \mathrm{bp}$ reads. The script for RNAseq analysis can be found in github (https://github.com/milospjanic/rnaSeqFPro). Briefly, QC of sequencing data was performed using fastqc. Then, STAR was used to map the reads to the reference genome (hg19). FeatureCounts from the Subread package was then used to count the number of reads mapped, and deseq 2 was used to generate the list of differentially regulated genes.

\section{Results}

JUN and TCF21 regulate CAD candidate causal gene expression in HCASMC

AP-1 and TCF21 are regulatory transcription factors in human coronary artery smooth muscle cells (HCASMC) in a number of CAD loci, including SMAD3 and $9 p 21$ $[20,33]$. To investigate the specific roles of AP-1 and TCF21 in regulating CAD gene transcription, we first focused our investigation on two candidate causal genes, CDKN2BAS and SMAD3 [33, 38]. siRNA knockdown of $J U N$ in HCASMC was associated with decreased expression levels of CDKN2BAS and SMAD3 (Fig. 1a, c, Additional file 1: Figure S1A). On the other hand, knockdown of TCF21 produced an opposite effect compared to JUN knockdown, producing increases in SMAD3 and CDKN2BAS levels (Fig. 1a, c, Additional file 1: Figure S1A). TCF21 overexpression produced results opposite to knockdown (Fig. 1a, c, Additional file 1: Figure S1B). Given the known anti-sense effect of 

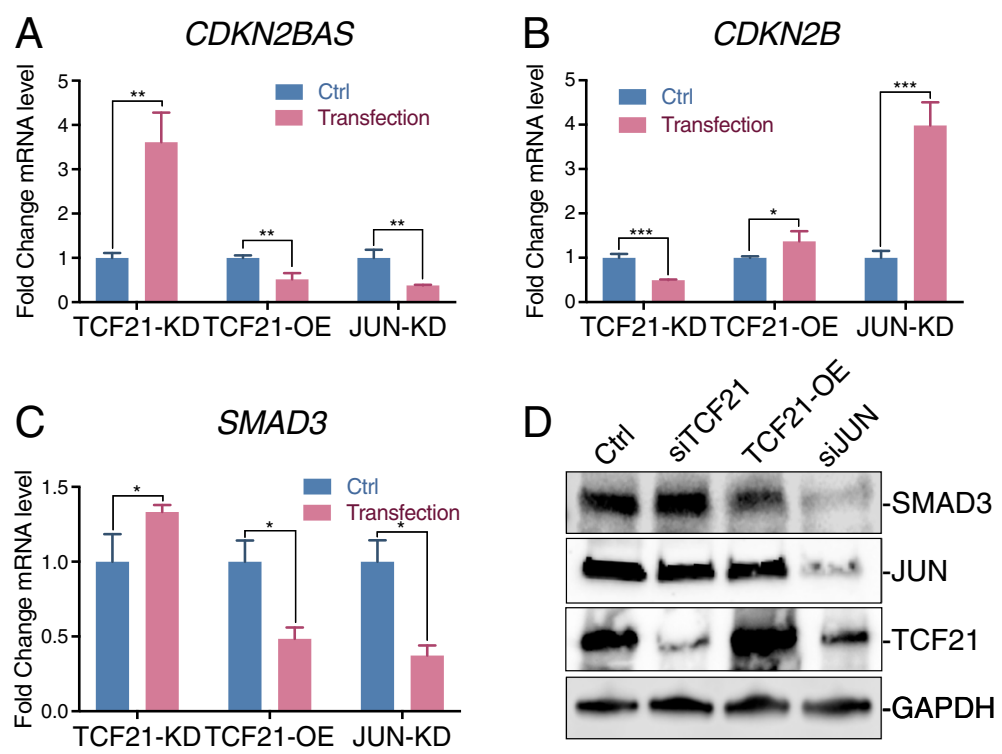

Fig. 1 JUN and TCF21 regulate expression levels of CAD genes SMAD3 and CDKN2BAS/CDKN2B in HCASMC. a HCASMC were transfected with JUN (JUN-KD), TCF21 (TCF21-KD), scrambled (Ctrl) siRNA molecules, or transduced with empty pWPI vector (Ctrl) or a human TCF21 CDNA clone (TCF21-OE) virus. The mRNA expression level of CDKN2BAS was evaluated by qPCR with ACTB normalization. b, c CDKN2B and SMAD3 mRNA expression levels were quantified under identical conditions in HCASMC (mean \pm SD, $n=3$ ). d SMAD3, JUN, and TCF21 protein levels were evaluated by western blot, with GAPDH as loading control

CDKN2BAS [45], changes in CDKN2BAS expression were mirrored with opposite effects for the mRNA level of CDKN2B (Fig. 1a, b). To confirm these gene expression changes, we investigated the protein level of SMAD3 by western blotting. The SMAD3 protein level decreased with JUN knockdown or TCF21 overexpression and increased with TCF21 knockdown, consistent with mRNA levels (Fig. 1d, Additional file 1: Figure S1C). These data suggest that AP-1 serves as a transcriptional activator, and TCF21 acts as a suppressor of the presumptive CAD causal genes CDKN2BAS and SMAD3.

\section{JUN increases H3K27ac level and chromatin accessibility to promote TCF21 binding in SMAD3 and CDKN2BAS CAD loci}

In our previous studies, we identified enhancer regions associated with SMAD3 and CDKN2BAS genes [33]. The presumptive causal variants located in these regions, rs17293632 in the SMAD3 enhancer and rs1537373 in the CDKN2BAS enhancer, are in close proximity to AP1 and TCF21 binding sites [2-4, 33, 35, 46, 47]. These variants are located in HCASMC ATACseq open chromatin and, by eQTL and allele-specific expression, have been linked to the expression of SMAD3,CDKN2BAS, or $C D K N 2 B$ [33, 34]. Thus, we designed two ChIP-qPCR primer sets for the $S M A D 3$ enhancer. One set of primers amplifies the SMAD3-1 region, containing the AP-1 binding motif which is disrupted by rs17293632, and the second set amplifies the SMAD3-2 region containing the
TCF21 binding motif which is $+505 \mathrm{bp}$ downstream from AP-1 binding site (Additional file 1: Figure S1D). However, AP-1 factors can also bind to the SMAD3-2 region to some degree as shown by enrichment for JUN ChIPseq reads (Additional file 1: Figure S1D), and although not evident with the genome browser settings shown, TCF21 can bind to SMAD3-1 with a small but significant number of TCF21 ChIPseq reads (Additional file 1: Figure S1D). Another primer set was designed to amplify the CDKN2BAS-CDKN2B enhancer, containing both AP-1 and TCF21 binding motifs downstream from rs1537373 (Additional file 1: Figure S1E). To better understand the mechanism of how AP-1 affects the transcription of CAD genes, we evaluated transcription factor binding and chromatin state in these loci in HCASMC by ChIP-qPCR. Compared with control siRNA transfection, JUN knockdown resulted in significantly decreased JUN binding in both SMAD3 (SMAD3-1 and SMAD3-2) and CDKN2BAS loci (Fig. 2a). Since JUN promotes the endogenous expression of TCF21 [20], we overexpressed TCF21 with lentivirus transduction to maintain the TCF21 expression level in JUN knockdown and control cells (Fig. 2b, Additional file 1: Figure S2A, S2B). With sustained TCF21 level in these cells, JUN knockdown still resulted in reduced TCF21 binding to these loci (Fig. 2c, Additional file 1: Figure S2A, S2B). Associated with JUN's decrease, histone H3K27 acetylation was also decreased (Fig. 2d). Lower H3K27ac levels resulted in decreased chromatin accessibility in these loci 


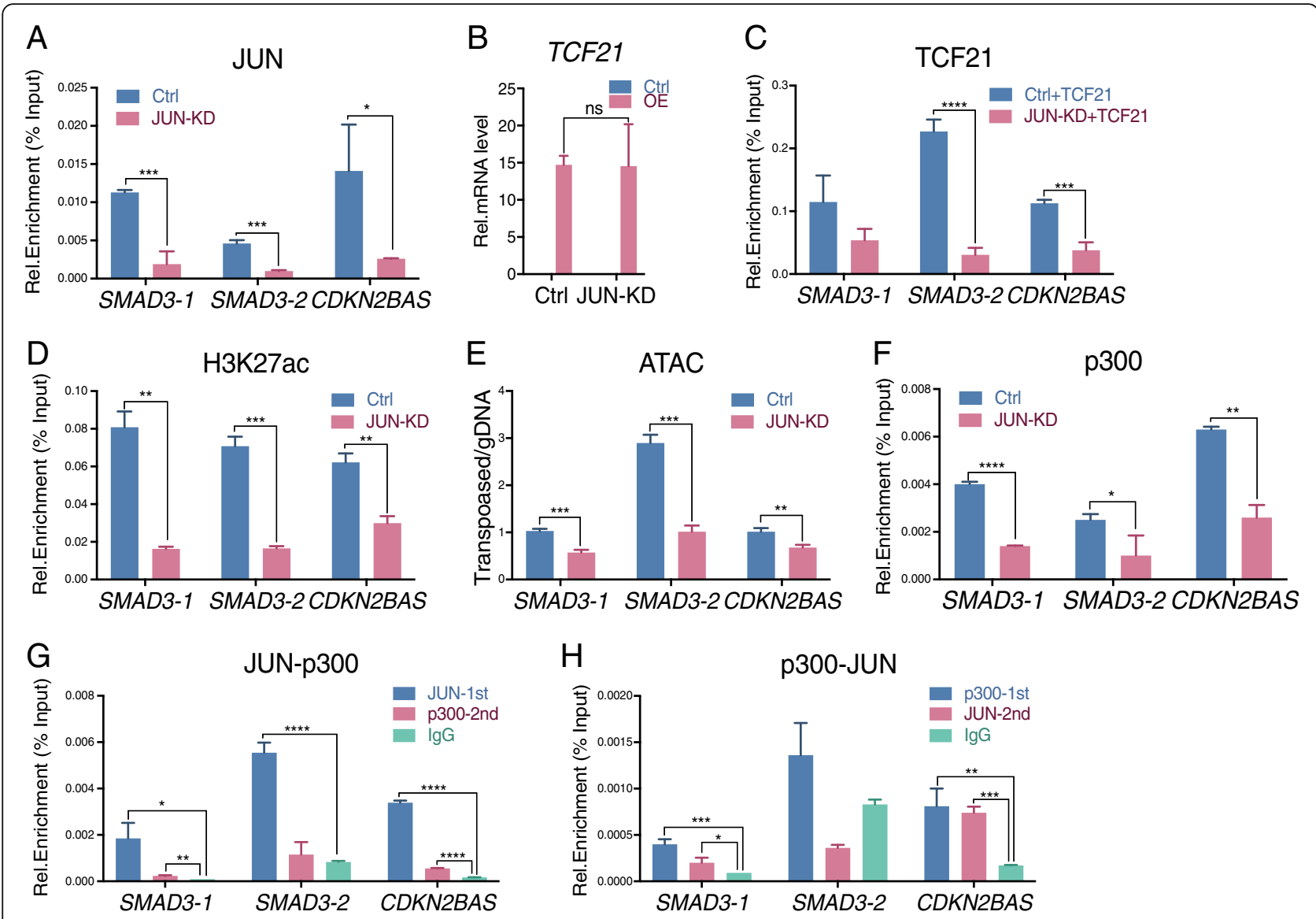

Fig. 2 JUN recruits p300, promotes H3K27ac histone modification, and increases chromatin accessibility and TCF21 binding. a-f ChIP-qPCR at SMAD3-1, SMAD3-2, and CDKN2BAS locus regions with input normalization in HCASMC. a JUN binding was evaluated under conditions of JUN (JUN-KD) and scrambled (Ctrl) siRNA transfections. $\mathbf{b}$ Validation of overexpressed TCF21 mRNA level under conditions of JUN-KD and Ctrl siRNA transfections by RT-qPCR. c pWl lentivirus expressed TCF21 binding was evaluated under conditions of JUN-KD or Ctrl siRNA transfection. $\mathbf{d}$ H3K27ac level was evaluated under conditions of JUN-KD or Ctrl siRNA transfection. e Chromatin accessibility assessed by ATAC-qPCR was evaluated under conditions of JUN-KD or Ctrl siRNA transfection. $\mathbf{f}$ p300 binding at SMAD3-1/SMAD32 and CDKN2BAS loci was evaluated under conditions of JUN-KD or Ctrl siRNA transfection. $\mathbf{g}$ Serial ChIP-qPCR with JUN first IP followed by p300 second IP. $\mathbf{h}$ Serial ChIP-qPCR with p300 first IP followed by JUN second IP $($ mean $\pm S D, n=3)$

(Fig. 2e). These data demonstrate that JUN binding in these candidate causal CAD loci leads to altered histone modification, chromatin accessibility, and recruitment of chromatin remodeling factors, as a potential mechanism of their effects on gene expression in HCASMC.

\section{JUN recruits EP300 to acetylate H3K27 and open chromatin in CAD loci}

We next investigated how AP-1 complex transcription factors regulate histone modification and chromatin state. In vitro purification studies have provided evidence that the AP-1 complex interacts with histone acetyltransferases (HATs), suggesting that EP300 (p300) may be involved in transcriptional regulation in HCASMC [48-50]. To test this hypothesis, we assessed whether p300 binding in SMAD3 and CDKN2BAS loci is dependent on JUN expression level. ChIP-qPCR studies showed p300 binding requires the presence of $\mathrm{JUN}$ as p300 binding is significantly decreased in the absence of JUN (Fig. 2f). Consistent with p300's role as a HAT, JUN overexpression increased H3K27ac level in the SMAD3 locus, which could be negated by knocking down $\mathrm{p} 300$ (Additional file 1 : Figure S2C, S2D). These studies demonstrated that JUN regulates H3K27ac of enhancers in a p300-dependent manner. Interestingly, although JUN effects on TCF21 binding can also be reversed by $p 300$ knockdown, $p 300$ knockdown alone cannot affect the TCF21 binding in the SMAD3 locus (Additional file 1: Figure S2E). These findings suggest that it is AP-1, not the histone modification enzymes, that plays a leading role in guiding the binding of other TFs, such as TCF21.

To further explore the relationship between AP-1 and HATs, we employed serial ChIP to study their interactions. With the first immunoprecipitation (IP) using 
JUN antibody, followed by a second IP using p300 antibody with the eluate from the first IP, we observed significant enrichment of both JUN and p300 on SMAD3-1 and $C D K N 2 B A S$ loci, while the SMAD3-2 locus showed enrichment for JUN and modest enrichment for p300 that was not significant (Fig. 2g). With the opposite direction serial ChIP, we also observed similar enrichment of p300 and JUN at SMAD3-1 and CDKN2BAS loci (Fig. 2h). Together, these data suggest that p300 is recruited by AP-1 to the CAD SMAD3 and CDKN2BAS loci, thereby facilitating JUN-mediated histone acetylation, chromatin accessibility, and binding of other transcription factors.

\section{TCF21 reduces H3K27ac level and chromatin accessibility} but not JUN binding at CAD loci

In contrast to AP-1, TCF21 appears to function primarily as a transcriptional repressor [22, 23], suggesting that its effect on transcription in CAD loci might be quite different from AP-1. This is consistent with other bHLH factors that oppose AP-1 actions [51]. To better understand how TCF21 modulates gene expression, we investigated the level of H3K27ac, chromatin accessibility, and JUN binding in the SMAD3 and CDKN2BAS loci under TCF21 siRNA knockdown or lentivirus overexpression in HCASMC. The ChIP-qPCR data from cells with TCF21 knockdown show that with decreased TCF21 binding in these loci (Fig. 3a), the H3K27ac levels and chromatin accessibility are increased (Fig. 3b, c). Decreased chromatin accessibility is observed at SMAD3-1 and SMAD3-2 with increased TCF21 expression which is consistent with the observed effects on H3K27ac status, although CADKN2BAS shows a slight and unexpected increase in H3K27ac when TCF21 binding is elevated by lentivirus (Fig. $3 \mathrm{a}-\mathrm{c}$ ). Interestingly, JUN binding in these loci is not significantly affected by reduced or elevated TCF21 binding (Additional file 1: Figure S3A). Combined with the JUN knockdown data, these findings suggest that JUN binding is upstream and likely precedes TCF 21 binding, with the AP- 1 complex serving as a pioneer factor that recruits TCF21 itself after binding DNA and modifying the chromatin environment, as has been characterized in the case of the glucocorticoid receptor [30]. These data are consistent with our previous observations that JUN activation
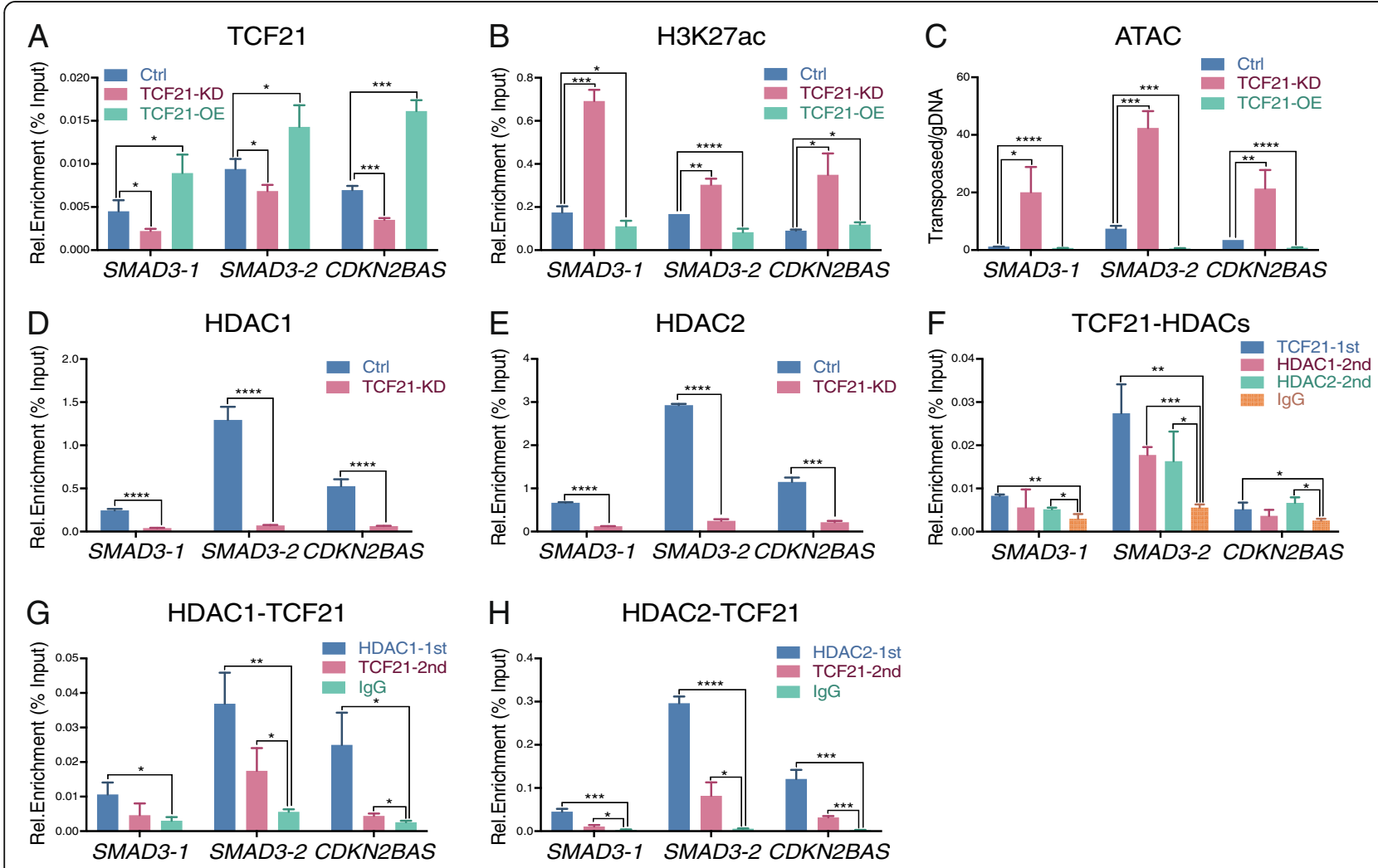

Fig. 3 TCF21 recruits HDACS 1 and 2, promotes deacetylation at H3K27ac, and decreases chromatin accessibility. All panels show ChIP-qPCR at SMAD3-1, SMAD3-2, and CDKN2BAS locus regions with input normalization in HCASMC. a TCF21 binding, b H3K27ac level, and $\mathbf{c}$ chromatin accessibility (ATAC-PCR) were evaluated under the conditions of TCF21 (TCF21-KD) or scrambled (Ctrl) siRNA transfections or lentivirus overexpression of TCF21 (TCF21-OE). d HDAC1 and e HDAC2 binding were evaluated by ChIP-qPCR with TCF21-KD. f Serial ChIP-qPCR with TCF21 first IP followed by HDAC1 or HDAC2 second IP. $\mathbf{g}$ Serial ChIP-qPCR with HDAC1 first IP followed by TCF21 second IP. $\mathbf{h}$ Serial ChIP-qPCR with HDAC2 first IP followed by TCF21 second IP (mean \pm SD, $n=3$ ) 
precedes TCF21 expression [20]. Together, these results indicate that TCF21 is a suppressor of the two CAD presumptive causal genes under study here, SMAD3 and $C D K N 2 B A S$, and suggest that the transcriptional inhibition by TCF 21 is possibly mediated through H3K27ac, but that this effect does not alter AP-1 binding.

\section{TCF21 recruits HDAC1 and 2 to deacetylate H3K27 at CAD} loci

It has been previously shown that TCF21 function in the androgen receptor pathway is histone deacetylase (HDAC) 1 dependent [52] and that it directly associates with HDAC2 [53], suggesting a possible mechanism for how TCF21 suppresses gene transcription in HCASMC. To answer this question, we evaluated both HDAC1 and HDAC2 binding in SMAD3 and CDKN2BAS loci in ChIP-qPCR experiments. These data showed a significant reduction in both HDAC1 and HDAC2 binding under TCF21 siRNA knockdown (Fig. 3d, e), along with decreased TCF21 binding level (Additional file 1: Figure S3B).

We further investigated the interaction between TCF21 and HDACs with serial ChIP experiments. With the first IP using TCF21 antibody, followed by the second IP using HDAC1 or HDAC2 antibodies from the elution of the first IP, we observed significant enrichment of both HDAC1 and HDAC2 at SMAD3 and
CDKN2BAS loci in HCASMC (Fig. 3f). In a second series of experiments, we performed serial ChIP in the reverse direction, first IP with HDAC1 or HDAC2 antibody and second IP with TCF21 (Fig. 3g, h). These data suggest that TCF21 recruits $\mathrm{HDAC} 1$ and HDAC2 to SMAD3 and CDKN2BAS genetic loci to deacetylate H3K27 and thereby suppress transcription.

\section{JUN and TCF21 co-localize at CAD enhancers and directly interact through protein-protein binding}

AP-1 and TCF21 binding sites are juxtaposed in the $S M A D 3$ and $C D K N 2 B A S$ loci (Additional file 1: Figure S1D, S1E), suggesting a possible direct interaction between these two factors. We employed serial ChIP, ChIP-western blotting, and co-IP experiments to investigate this hypothesis. First, serial ChIP studies provided evidence for chromatin-dependent interaction at the SMAD3 and CDKN2BAS loci. Either with the first IP using a JUN antibody, followed by a second IP using TCF21 antibody with the eluate of the first IP (Fig. 4a), or performing the IPs in the opposite direction (Fig. 4b), co-enrichment of TCF21 and JUN were detected at the SMAD3 and CDKN2BAS loci in HCASMC. Second, we performed ChIP-western blotting experiments with formaldehyde crosslinked HEK293 cell line transfected with plasmids encoding myc-tagged TCF21 and native
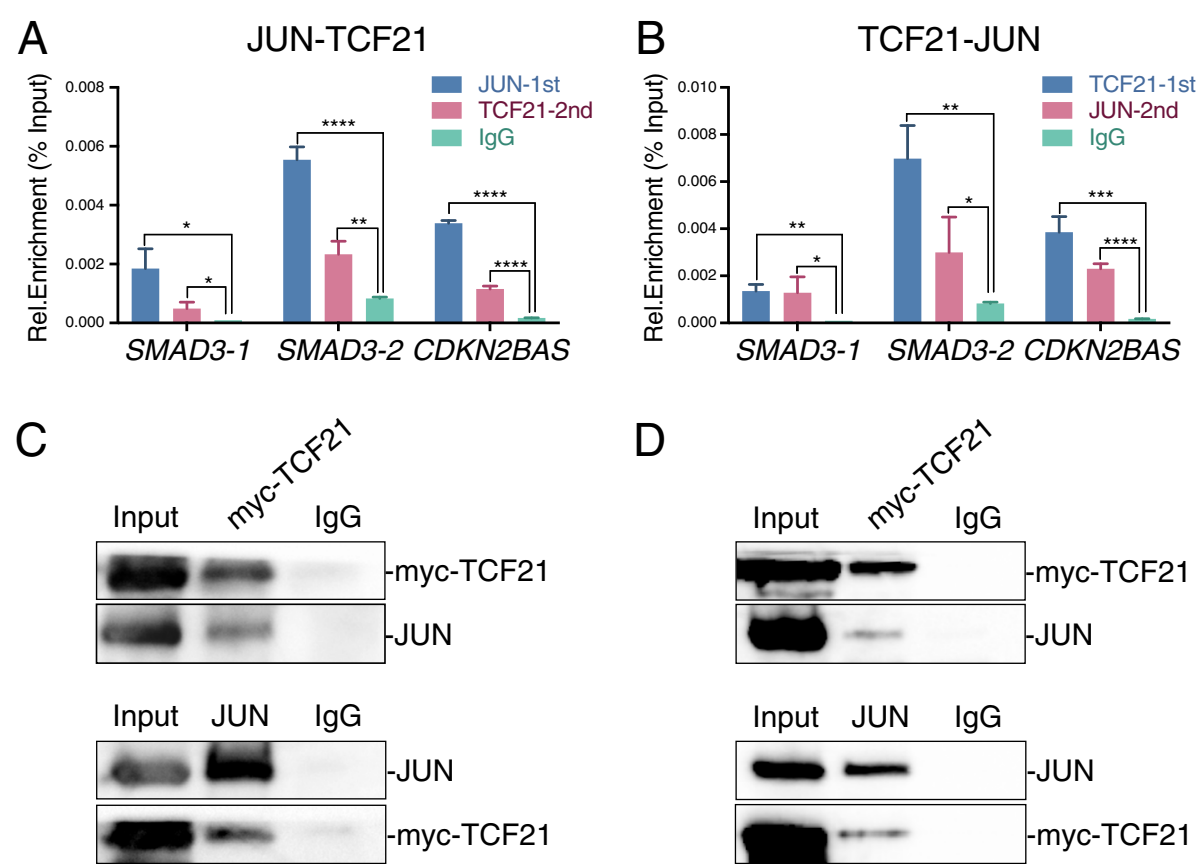

Fig. 4 JUN and TCF21 co-occupy chromatin at SMAD3 and CDKN2BAS loci. a Serial ChIP-qPCR with JUN first IP followed by TCF21 second IP. b Serial ChIP-qPCR with TCF21 first IP followed by JUN second IP (mean \pm SD, $n=3$ ). c Interaction between TCF21 and JUN on chromatin was evaluated by ChIP-western blotting, IP with myc-TCF21 (top) followed by JUN western, or with reverse conditions (bottom). Myc-tagged TCF21 and non-tagged JUN expression constructs were transfected into HEK293 cells. d Co-immunoprecipitation of TCF21 and JUN with IP of myc-TCF21 followed by JUN western (top) and reverse conditions (bottom). Myc-tagged TCF21 and non-tagged JUN were transfected into HEK293 cells 
JUN. IP was performed with myc-tag or JUN antibodies in the ChIP buffers followed by reversal of crosslinking and western blotting (Fig. 4c). These data, from IP performed in both directions, provide additional evidence for co-localization of AP1 and TCF21 in enhancer regions of these two CAD loci. Finally, we transfected TCF21 and $J U N$ plasmids into HEK293 cells and performed direct co-IP experiments to look for protein-protein interaction of these factors. The results showed that JUN can be pulled down with myc-tagged TCF21 IP, and myc-tagged TCF21 also identified in JUN IP (Fig. 4d). Taken together, these data provide compelling support for AP-1 and TCF21 binding together at sites in the genome and directly interacting via protein-protein binding.

\section{JUN and TCF21 binding is co-localized genome-wide and these factors regulate $\mathrm{H} 3 \mathrm{~K} 27 \mathrm{ac}$ levels in the shared loci}

We have previously reported ChIPseq data for TCF21 and JUN in HCASMC [23] and documented a significant overlap of the binding regions in CAD-associated loci [33]. To further investigate the co-localization of JUN and TCF21 binding genome-wide, and the resulting impact of their binding on local epigenomic features, we have mapped H3K27ac histone modifications in conjunction with JUN and TCF21 ChIPseq under knockdown and control conditions in HCASMC.

First, we compared the binding of native levels of JUN and TCF21, along with H3K27ac modifications, in the control ChIPseq datasets. We found co-localization of TCF21 and H3K27ac enrichment centered on total 85,695 JUN peaks, compared with the negative control TF HNF1A. Visualization of this co-localization with a density plot for normalized ChIPseq peaks showed H3K27ac modification overlapping and flanking the JUN peaks, along with TCF21 overlap as previously described (Fig. 5a) [33]. Focusing on a total 42,490 TCF21 peaks revealed co-localization of JUN binding and H3K27ac marked regions. A density plot showed more prominent overlap with JUN binding and H3K27ac mapping with a similar pattern of co-localization (Fig. 5b). We also plotted position weight matrix (PWM) scans for predicted binding at JUN and TCF21 peaks, which showed similar co-localization of the TFs (Additional file 1: Figure S4A, S4B). To quantify the number of overlapping JUN and TCF21 peaks, we intersected these two groups of ChIPseq peaks, identifying 12,033 TCF21 peaks that directly overlap with JUN peaks $(P<1.41 \mathrm{e}-91$, Fig. $5 \mathrm{c})$. The overlapped TCF21 peak number increased rapidly with the distance from JUN, and more than 70\% of TCF21 peaks $(30,120$ out of 42,490$)$ are found less than $10 \mathrm{~kb}$ from JUN binding sites (Fig. 5c), suggesting relative proximity of their binding genome-wide and possible regulatory interactions.
Co-localization of binding was verified by investigating the binding site distance from the control factor HNF1A, a TF that is functional primarily in the liver, and has different binding features from TCF21 and AP-1. We intersected DNaseI open chromatin data for HepG2 liver cells from ENCODE and our ATACseq data for HCASMC to get the common open chromatin regions in these two cell types, and analyzed the closest distances of binding sites for these various factors. The histogram of distance shows no significant difference between the spacing of TCF21 from JUN, JUN from TCF21, or TCF21 from HNF1A in the common open chromatin regions (Additional file 1: Figure S4D). However, if we exclude the common open regions of ENCODE from HCASMC open regions to obtain HCASMC-specific open chromatin regions, the median distance between binding sites of TCF21 and JUN is $12,781 \mathrm{bp}$, while that of TCF21 and HNF1A is 78,559 bp (Additional file 1: Figure S4E), suggesting that the co-localization of TCF21 and JUN is smooth muscle cell type-specific.

Since we have shown that AP-1 and TCF21 can both modulate $\mathrm{H} 3 \mathrm{~K} 27 \mathrm{ac}$ histone modification in CAD loci in HCASMC, we were interested in mapping and comparing loci across the genome where these TFs regulate H3K27ac status in HCASMC. Thus, we performed H3K27ac ChIPseq under TCF21 shRNA or JUN siRNA knockdown, obtaining 24,065 and 24,883 total peaks under these conditions, respectively. Heatmap distributions of JUN peaks showed genome-wide enrichment of TCF21 binding, with enrichment of H3K27ac showing a flanking pattern (Fig. 5d) as in the density plot, and diminished signal in the JUN knockdown condition. Similar heatmaps of TCF21 binding showed enrichment for JUN co-localization, with more widely distributed H3K27ac enrichment that is more pronounced with TCF21 knockdown (Fig. 5e). To quantify the change in $\mathrm{H} 3 \mathrm{~K} 27 \mathrm{ac}$ status genome-wide, we evaluated the average enrichment levels on H3K27ac peaks found in the sequencing data. Compared with scrambled control, H3K27ac level in JUN siRNA knockdown showed a change in peak length distribution with shorter length peaks with $J U N$ knockdown and longer peaks with TCF21 knockdown (Fig. 5f, g) (Wilcoxon tests, both $P<2.2 \mathrm{e}-16)$.). In addition, JUN siRNA knockdown showed a modest but significant reduction in H3K27ac peak height for all peaks (Fig. 5h), while the level in TCF21 shRNA knockdown showed a significant increase (Fig. 5I).

Further, we identified loci where H3K27ac is regulated by both JUN and TCF21 in HCASMC. With JUN knockdown, we obtained 10,882 decreased and only 391 increased H3K27ac peaks compared with control. On the other hand, 42 decreased and 14,234 increased H3K27ac peaks were found in TCF21 knockdown. 

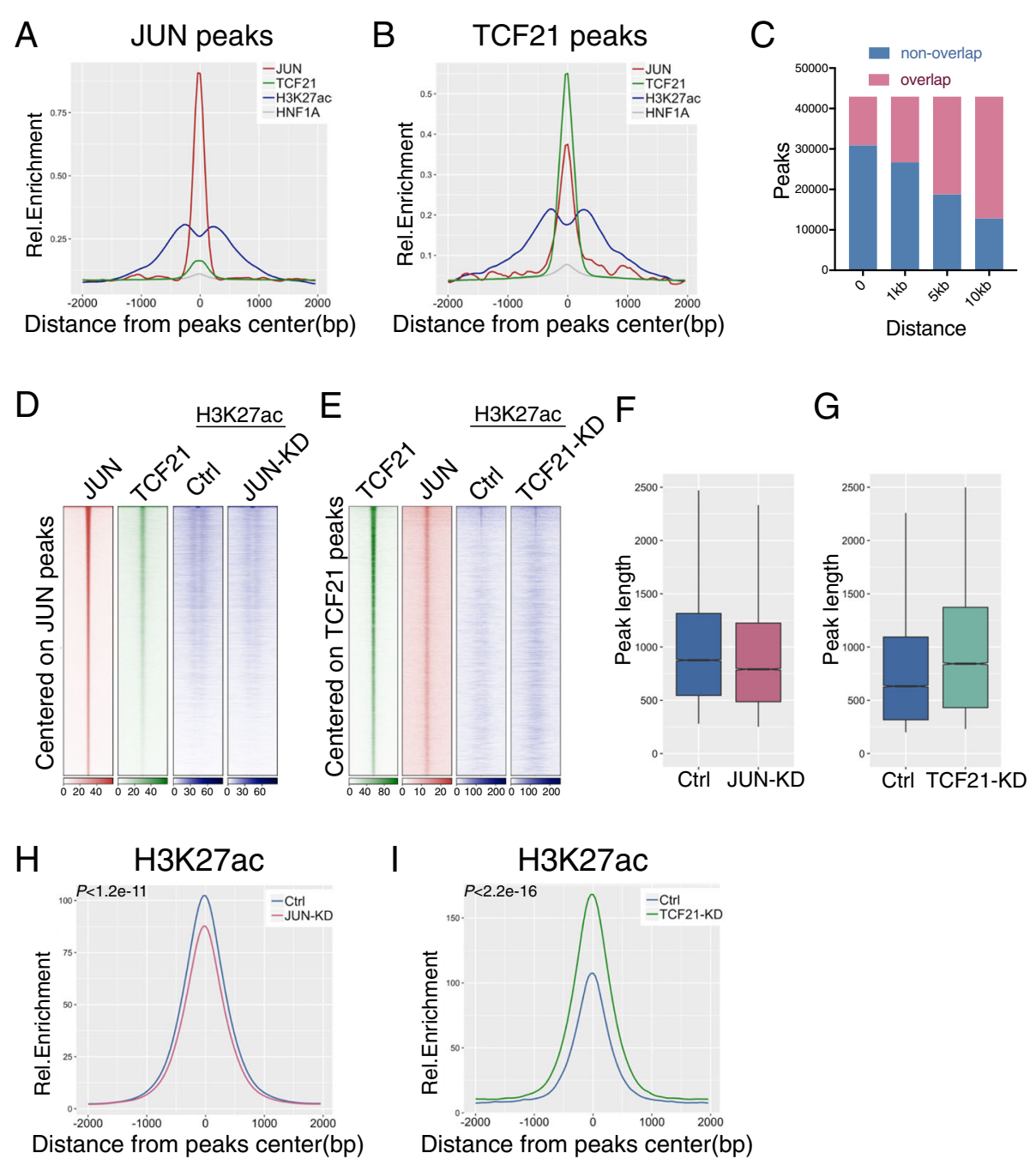

Fig. 5 JUN and TCF21 co-localize genome-wide and regulate H3K27ac chromatin modification. a JUN ChIPseq peaks and b TCF21 peaks were extended $+/-2 \mathrm{~kb}$ from summit, and a density plot created for RPM normalized enrichment levels of JUN, TCF21, and H3K27ac ChIPseq in HACSMC, along with control transcription factor HNF1A. c The number of TCF21 peaks overlapped with JUN binding for distances between peaks less than $1 \mathrm{~kb}, 5 \mathrm{~kb}$, and $10 \mathrm{~kb}$. d Heatmap distribution of ChIPseq data for JUN, TCF21, and H3K27ac with control (Ctrl) or JUN knockdown (JUNKD) centered on JUN peaks, or e TCF21 knockdown (TCF21-KD) centered on TCF21 peaks within a 4-kb window. $\mathbf{f}$ Peak length distribution of Ctrl or JUN-KD, and $\mathbf{g}$ Ctrl or TCF21-KD H3K27ac ChIPseq in HCASMC (box plots with Wilcoxon tests, $P<2.2 \mathrm{e}-16$ ). $\mathbf{h}$ H3K27ac ChIPseq enrichment level of Ctrl or JUN-KD, and $\mathbf{i}$ Ctrl or TCF21-KD, centered on all H3K27ac peaks with +/- $2 \mathrm{~kb}$ extension, normalized by RPM

With a more stringent $q$ value cutoff $(q<1 \mathrm{e}-10$ for TCF2 1 and $q<1 \mathrm{e}-5$ for JUN), chosen to give equivalent numbers of peaks, we found 7017 peaks with H3K27ac increased with JUN knockdown and 6026 peaks with H3K27ac decreased with TCF21 knockdown. Out of these, 3093 peaks were directly overlapping (Additional file 1: Figure S4F). We also intersected those 3093 overlapping H3K27ac peaks with TCF21 and JUN ChIPseq peaks, showing that 749 peaks overlap with TCF21, 699 with JUN, and 224 with both (Additional file 1: Figure S4G). Therefore, 1124 H3K27ac peaks overlap with at least one of the two transcription factor ChIPseq sites, suggesting that more than one third of these sites are directly regulated by TCF21 and JUN, while two thirds are indirectly regulated by TF downstream of TCF21 and JUN. These data indicate that H3K27ac level is regulated at the genome-wide level by AP-1 and TCF21, thus supporting our ChIP-qPCR data and suggesting a genomewide epigenomic interaction of these factors. Overall, changes in H3K27ac level with JUN knockdown were not as pronounced as those seen for TCF21 knockdown, possibly due to the fact that there are a number of bZip factors that can bind and regulate H3K27ac at AP-1 sites in addition to JUN, leading to a smaller effect size.

Finally, we employed Genomic Regions Enrichment of Annotations Tool (GREAT) to assign the common 3093 H3K27ac regions to genes, and this collection of 2580 target genes were used in gene ontology analysis with 
DAVID. Significant terms identified by the Biological Process analysis included VEGF, TGF $\beta$ and EGF signaling pathways, cell adhesion, migration, and proliferation, which are highly relevant to smooth muscle cellular functions (Additional file 1: Figure S4H, Additional file 2: Table S1). Significant KEGG terms included VEGF, TGF $\beta$ and Hippo pathways, focal adhesion, and cell cycle (Additional file 1: Figure S4I, Additional file 2: Table S1). These data suggest that AP-1- and TCF21regulated $\mathrm{H} 3 \mathrm{~K} 27 \mathrm{ac}$ loci are highly associated with basic HCASMC functions that are relevant to CAD pathophysiology.

\section{JUN and TCF21 regulate chromatin accessibility in HCASMC}

Open chromatin regions in HCASMC are enriched for CAD-associated loci and GWAS variants $[19,33]$. To investigate the epigenetic implications of these observations, we employed ATACseq to generate epigenomic profiles in HCASMC with JUN knockdown, TCF21 knockdown, and TCF21 overexpression. With TCF21 knockdown, we obtained 54,298 increased open chromatin regions and 2218 decreased regions; while 9932 decreased regions were found with TCF21 overexpression, all with $q<0.01$. Since TCF21 is mainly identified as a transcriptional suppressor [22, 44], we intersected the 54,298 increased regions with knockdown and the 9932 decreased regions with overexpression, identifying 5063 overlapped high confidence TCF21 targets. Similarly, we intersected 110,373 and 109,143 decreased regions identified with JUN knockdown by two different shRNAs, shJUN-1 and -2, thus identifying 37,352 JUN high confidence ATACseq targets with $q<1 \mathrm{e}-10$ and fold change $<0.5$. There were 2587 overlaps within the TCF21 and JUN target regions, which we identified as loci regulated by the two TFs with high confidence $(P<6.2 \mathrm{e}-83$, Fig. 6a). We also integrated these loci with ChIPseq profiles for JUN and TCF21 binding as well as those for H3K27ac modifications. A large portion of the $2587 \mathrm{JUN}$ and TCF21 ATACseq targets overlap with TCF21 and JUN peaks; 1043 with TCF21, 2111 with JUN, and 885 with both (Fig. 6b). The H3K27ac peaks were also highly enriched in the open chromatin regions of $J U N$ or TCF21 knockdown, with $\sim 90 \%$ overlap with ATAC peaks (Additional file 1: Figure S5A, S5B). Further, we centered the 2587 loci for heatmap clustering, showing the co-localization of these open chromatin regions with JUN and TCF21 binding, but not with the negative control HNF1A (Fig. 6c). The enrichment levels of ATACseq on these loci are reduced in JUN knockdown while they are upregulated in TCF 21 knockdown and downregulated in TCF21 overexpression (Fig. 6c), which is consistent with the regulated peak numbers above. We also discovered several AP-1 and TCF21/TCF12 binding motifs using the HOMER "known" motif analysis on these 2587 loci with low $P$ values (Additional file 1: Figure $\mathrm{S} 5 \mathrm{C}$ ). These analyses provide a map of genomic regions targeted by AP-1 and TCF21 in HCAMSC.

To investigate the genes in the open chromatin regions where JUN and TCF21 are co-localized, we assigned those loci to genes using GREAT. At the identified 936 genes, chromatin accessibility is downregulated by TCF21 and upregulated by JUN, especially around the TSS (Fig. 6d). At the SMAD3 and CDKN2BAS enhancers studied above, we observed pronounced chromatin accessibility differences with $J U N$ or TCF21 disruptions (Fig. 6e). In addition, smooth muscle cell marker genes such as ACTA2 and MYH11 were regulated with similar directionality (Additional file 1: Figure S5D). At the genome level, GO analysis identified a large number of the 936 genes to be significantly enriched in smooth muscle cell functions and CAD-associated terms, including vasculogenesis, angiogenesis, cell adhesion, and proliferation in biological processes; myocardial infarction, cardiovascular diseases, and coronary artery calcification in GAD enrichment; ECM and cell adhesion in KEGG pathways (Fig. 6f, g, Additional file 1: Figure S5E, Additional file 3: Table S2, Additional file 4: Table S3). The change in chromatin accessibility results in differential expression levels of those genes, $\sim 2 / 3$ of which are upregulated in mRNA level with TCF21 siRNA knockdown (Additional file 1: Figure S5F, Additional file 4: Table S3). In summary, these data reveal that AP-1 and TCF21 regulate chromatin modification and accessibility genome-wide in loci that regulate fundamental SMC processes; some of which are important mediators of CAD risk.

\section{CAD-associated variants are enriched in open chromatin regions regulated by JUN and TCF 21}

Given that the variation in chromatin accessibility and TF binding is a dominant mechanism of variation in gene expression, we were interested to identify and experimentally validate variants located in the 2587 regions of the genome that are regulated by AP-1 and TCF21 in HCASMC (Fig. 6a). First, we intersected those open chromatin regions with the GTEx Coronary Artery (CA) eQTL public variant database [54], as well as eQTLs we have mapped in HCASMC $(q<0.05)$ [19], and CARDIoGRAMplusC4D CAD [43]-associated SNPs $(P<0.05)$. There were 491 GTEx CA and 643 HCASMC eQTLs, and 857 CARDIoGRAMplusC4D SNPs located in these loci (Additional file 1: Figure S6A) [2, 4, 19, 55]. In particular, 643 HCASMC eQTLs were associated with 193 genes, which were enriched in CAD-associated terms in GO analysis [19]. Identified terms are quite similar to those found in AP-1- and TCF21-targeted loci (Additional file 1: Figure S6B, C, D, Additional file 5: Table S4). The expression level of these genes was also 


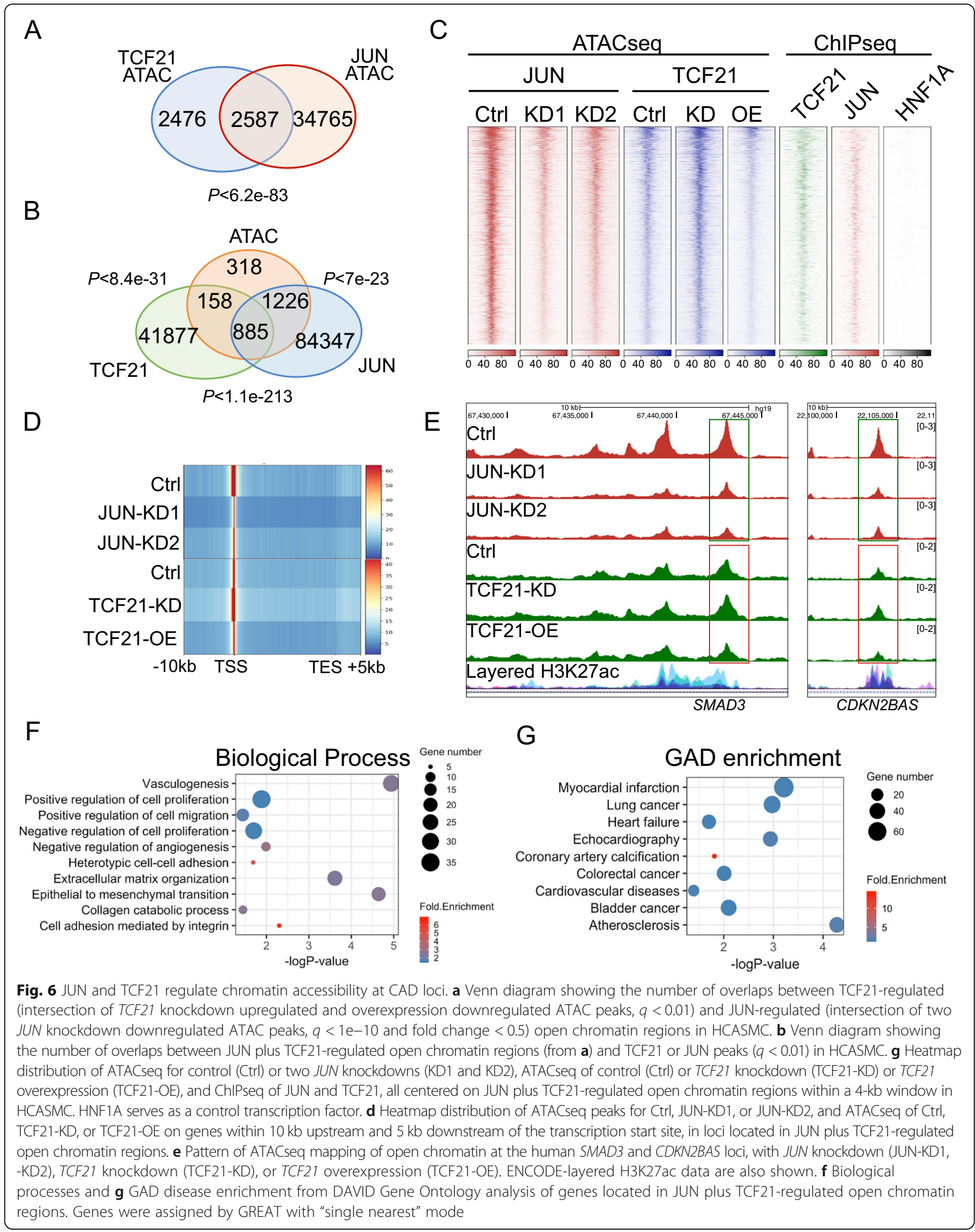


investigated in a previous TCF21 knockdown RNAseq study [22], showing that $>70 \%$ differentially expressed genes are elevated at the transcriptional level (Additional file 1: Figure S6E). With a second approach, employing the GWAS catalog with CARADIoGRAM +C4D data added, we intersected all GWAS variants within AP-1 plus TCF21-targeted regions of open chromatin; the results show that significant (low $P$ values) and highly enriched (high fold change) GWAS CAD SNPs were found in those regions (Additional file 1: Figure S6F). Further, we overlapped the JUN and TCF21 intersected peaks, (Additional file 1: Figure S6G), total TCF21 peaks (Additional file 1: Figure $\mathrm{S} 6 \mathrm{H}$ ), and total JUN peaks (Additional file 1: Figure S6I) with GWAS catalog SNPs. CAD-associated terms were highly enriched in overlapped regions of the two ChIPseq datasets compared to the individual datasets. Terms "CARDIoGRAM +C4D," "Coronary Artery Calcification," and "Coronary Artery" showed a 3-6-fold enrichment in the overlapping data analysis while JUN, and TCF21 alone had only 1-3-fold enrichment.

\section{Allele-specific binding of JUN and TCF21 leads to a transcriptional imbalance of presumptive CAD causal genes}

With these data regarding the role of AP-1 and TCF21 in genome-wide regulation of epigenetic features in CAD loci, we wanted to investigate the effect that such features have on allele-specific TF binding and CAD gene expression. In these experiments, we employed HCASMC from individuals heterozygous for candidate CAD regulatory SNP rs17293632 at SMAD3 that localize to a validated canonical AP-1 site in this gene [33]. The native $\mathrm{C}$ allele that is consistent with AP-1 factor binding was found to be associated with allelic expression imbalance of SMAD3 as detected by qRT-PCR, along with evidence of allelic differences in chromatin accessibility as reflected by ATACseq read counts, allele-specific binding of JUN, TCF21, and H3K27ac as identified with haploChIP studies (Fig. 7a).

To more formally address this question, we conducted follow-up studies in cells with CRISPR/Cas9 genome editing of the SNP rs17293632 in HEK293 cells. A series of edited lines were identified by genomic sequencing. A motif deleted (DEL) line and a single base-pair alteration line (ALT) which edited the $\mathrm{C}$ to $\mathrm{T}$ allele showed decreased SMAD3 expression compared to the native allele (Fig. 7b). These lines were used in ChIP-qPCR experiments which showed that motif deletion causes a significant reduction of JUN binding at rs17293632, as did the single nucleotide alteration (Fig. 7c). The decreased JUN binding can be observed at the edited rs17293632 loci but not with the native rs1537373 CDKN2BAS variant. The JUN-dominant negative factor
A-FOS can reduce the binding on both loci, showing that the reduction of JUN binding is caused by site-specific genome editing (Fig. 7c). We also investigated TCF21 binding and the chromatin state at these loci. Since no endogenous TCF21 expression was detected in HEK293 cells, we overexpressed TCF21 in these lines using lentivirus transduction. As expected, decreased JUN binding level resulted in reduced TCF21 binding, H3K27ac level, and chromatin accessibility at rs17293632 but not at rs1537373 (Fig. 7d-f). These data demonstrate that CAD variants can lead to allele-specific TF binding and imbalanced expression level of regulated genes.

\section{Discussion}

In conjunction with previously published data, the data presented here indicate that there are multiple mechanisms by which AP-1 factors may regulate transcription in CAD-associated loci and thus a disease risk. First, there are now several examples of AP-1 binding sites which are altered by causal variation, leading to differences in gene expression. An AP-1 cognate binding site in an intron of the SMAD3 gene has been shown to regulate expression of this gene and implicated as causal for both CAD and autoimmune diseases [20, 32, 34, 35, 56]. Also, causal variation at two CAD-associated alleles of TCF21 disrupts canonical AP-1 binding sites, which binds AP-1 factors and promotes expression of TCF21 [20]. A second mechanism, delineated by work presented here, provides compelling evidence that AP-1 may regulate expression of nearby TFs or chromatin regulatory features that are causal in disease loci by altering nearby epigenetic features and chromatin accessibility, i.e., through pioneer epigenetic functions (Fig. 8). This mechanism can also be extended to include the situation presented here whereby AP-1 regulates the binding and function of another functional TF such as TCF21, that in turn regulates the causal transcriptional mechanism. Finally, AP-1 factors are well known to interact with bHLH factors such as MYOD [57], and this fact in conjunction with evidence presented here for TCF21-AP-1 interaction suggests that AP-1 may modulate binding of other TFs through direct protein-protein interaction.

These data add to our understanding of how TCF21 affects CAD gene expression and function. TCF21 has a developmental role in coronary smooth muscle, specifically suppressing the expression of SMC lineage markers through transcriptional regulation/suppression (e.g., ACTA2) and possibly affecting the myocardin-SRF complex which serves as a master regulator in this cell type. Loss of TCF21 leads to increased SMC differentiation of CASMC. Reactivation of TCF21 may have a similar role in SMC in disease, promoting dedifferentiation and migration, which is protective in this setting, based on directionality from human genetics studies [21, 22]. In 


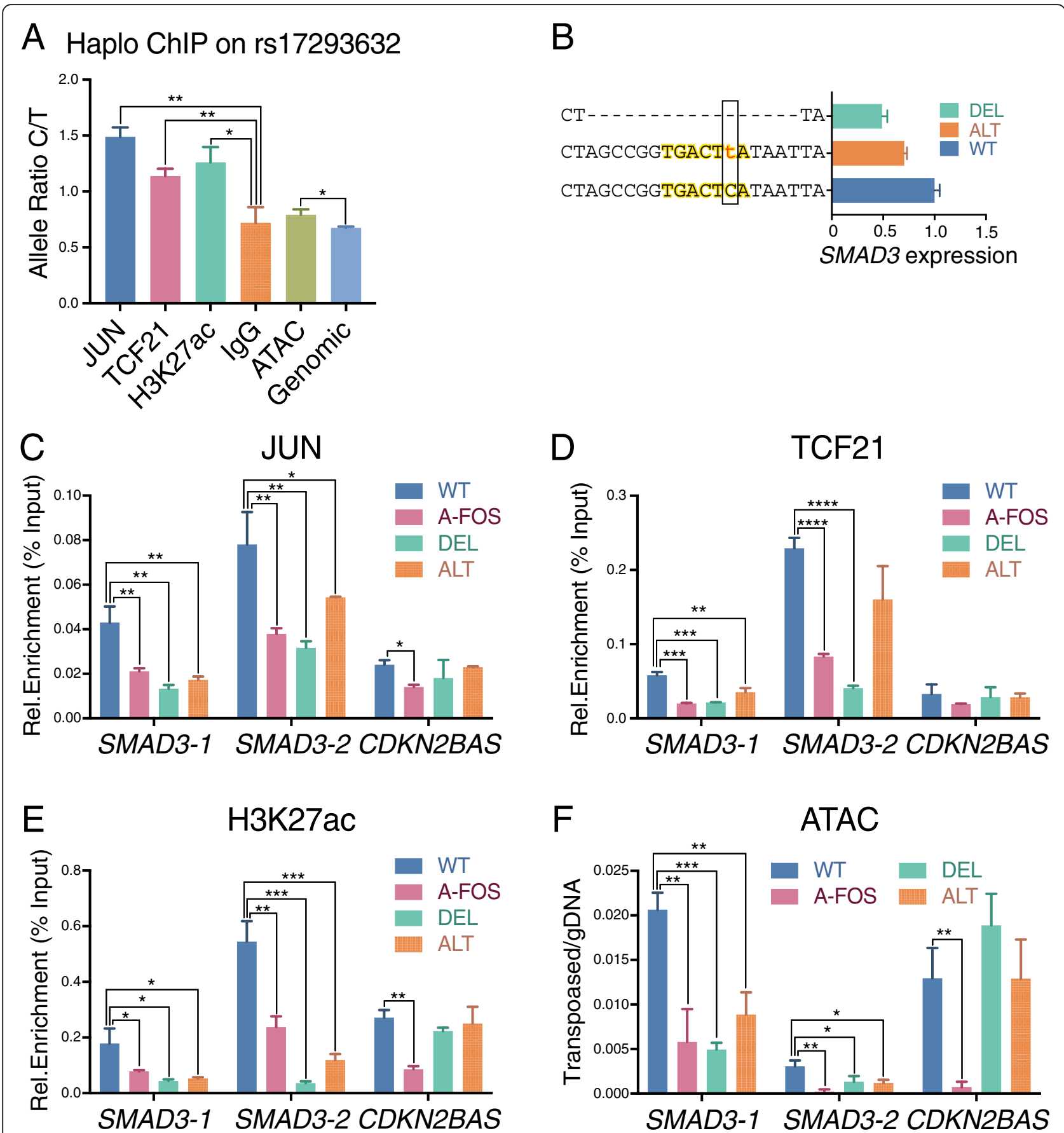

Fig. 7 Allele-specific binding of JUN, TCF21, and H3K27ac enrichment at the SMAD3 locus. a HaploChIP of JUN, TCF21, H3K27aC, and ATAC at rs 17293632 was evaluated by qPCR with allele-specific TaqMan probes in heterozygous HCASMCs. Enrichment was normalized with input, and data was shown as C/T ratio. b SMAD3 expression levels in wild type (WT), deletion (DEL), or converted (ALT) HEK293 CRISPR/Cas9 edited cell lines as detected by qPCR. The genomic sequence of CRISPR edits are shown for corresponding cell lines. c ChIP-qPCR of JUN, d TCF21, e H3K27ac, and $\mathbf{f}$ ATAC-qPCR showing the differential transcription factor binding, H3K27ac modification, or ATACseq open chromatin at SMAD3-1, SMAD3-2, and CDKN2BAS locus regions for WT or CRISPR HEK293 cell lines. Dominant negative A-FOS was expressed as a positive control of JUN inhibition, and all data normalized versus input (mean $\pm \mathrm{SD}, n=3$ ) 


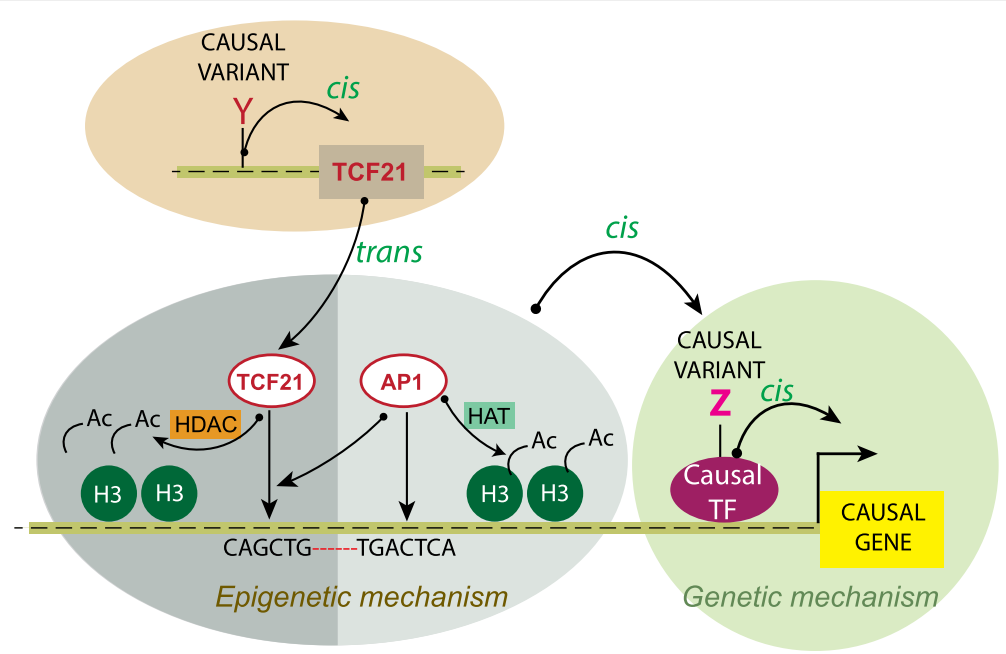

Fig. 8 Mechanism of TCF21 and AP-1 epigenetic interactions in the context of CAD-associated genetic loci. TCF21 is a bHLH transcription factor associated with CAD as depicted here due to allelic variation in causal variants $(Y)$, and its transcriptional regulatory function accounts for the attributable genetic risk at the 6q23.2 locus (brown oval). TCF21 binding is enriched in other CAD loci, where it interacts with AP-1 factors (JUN) that co-localize at these sites (gray oval). JUN promotes recruitment of HAT p300 to promote H3K27ac histone acetylation and open chromatin to recruit TFs, including TCF21, which in turn recruits HDACs 1 and 2 that function to oppose AP-1 effects. These epigenetic effects contribute in cis to the regulation of expression of the causal gene through alteration in the binding of the causal TF through CAD-associated variant Z (green circle) or other mechanisms of disease at this locus. Such interactions likely contribute to attributable genetic risk at both the TCF21 and downstream loci

addition to its capacity to regulate the transcriptional effect of the causal variant in the CAD locus, these data show that TCF21 has another mechanism of effect, which works through local epigenetic modification to regulate access of TFs and chromatin regulators to modulate gene expression. Further, these studies suggest that TCF21 can serve as a lineage partner for AP-1, similar to what has been shown for AP-1 interactions with PU.1 in macrophages, and ETS and GATA factors in endothelial cells where AP-1 promotes cell-restricted enhancer function $[28,58]$.

One important question that arises from these studies is the nature of the TCF21-AP-1 interaction in terms of CAD gene expression and disease risk. AP-1 is activated and binds to the SMAD3 and CDKN2BAS CAD loci, recruiting HAT activity and thus promoting an open chromatin configuration (Fig. 8). This promotes TCF21 binding, which in turn recruits HDACs, thus setting up a competition for the status of local chromatin configuration. The best explanation for these findings is that they represent a counter-regulatory pathway, with independent upstream epigenetic pathways regulating one or the other of these factors, and overall disease versus risk being determined by the cellular milieu in the vascular wall. It is important to note that the balance of regulation of promotion versus inhibition of transcription is more complex than just TCF21-AP-1 interactions, as these factors also likely regulate binding of CTCF, CEBP, and other TFs that bind in this region through their epigenetic effects. Importantly, for the SMAD3 and CDKN2BAS presumptive causal genes, TCF21 inhibits disease-promoting effects of AP-1 on the risk alleles at these two loci $[19,33]$. The same type of relationship has been characterized at CAD loci where TCF21 interacts with SMAD3, with the binding of both of these factors co-localizing genome-wide in a number of CAD loci [37]. That interaction is also primarily competitive, with TCF 21 blocking SMAD3 binding through epigenetic modulation. The effects of SMAD3 expression are opposite to TCF21 and promote disease risk, whereas AP-1 can be both protective through the promotion of TCF21 expression, and risk promoting through increasing SMAD3 expression [37].

While these studies highlight the interaction of AP-1 factors with TCF21 in loci that are linked to CAD, they likely represent a mechanism that is applicable to other human diseases. For instance, the mapped loci showing co-localization of AP-1 and TCF21 are enriched for genes that contribute to VEGF, TGF $\beta, E G F$, Hippo, focal adhesion, and cell cycle biological processes (Additional file 1: Figure $\mathrm{S} 4 \mathrm{H}$ ). These terms are well known to associate with various forms of cancer, and the data presented here are consistent with both the involvement of AP-1 and TCF21 interactions in the biology of the tumor cell, since TCF21 is a well-known tumor suppressor [59], as well as the angiogenic processes that support tumor growth and expansion [60,61].

\section{Conclusions}

These data characterizing the TCF21-AP-1 counterregulatory pathway in $\mathrm{CAD}$ loci suggests that the epigenetic 
landscape in disease loci is more complex than previously thought and that part of the attributable risk for CAD-associated transcription factors such as TCF21 may be due in part to their functions in trans, mediated by epigenetic effects at other CAD loci across the genome. The risk that resides in each disease locus would thus be a combination of the local mechanism involving the causal allele and gene, as well as that due to modulation of the epigenome by TFs such as TCF21 (Fig. 8).

\section{Additional files}

\section{Additional file 1: Supplemental figures and related figure legends.} (PDF $2496 \mathrm{~kb}$ )

Additional file 2: Table S1. Biological processes and KEGG pathways from DAVID Gene Ontology analysis of JUN-TCF21 regulated H3K27ac genes. (XLSX $16 \mathrm{~kb}$ )

Additional file 3: Table S2. GO analysis of genes in or nearby the open chromatin regions where JUN and TCF21 are co-localized. (XLSX $15 \mathrm{~kb}$ )

Additional file 4: Table S3. Genes in or nearby the open chromatin regions where JUN and TCF21 are co-localized, genes assigned by GREAT. (XLSX $22 \mathrm{~kb}$ )

Additional file 5: Table S4. Biological processes, KEGG pathways, and GAD disease enrichment of DAVID Gene Ontology analysis of HCAMSC eQTL target genes located in JUN plus TCF21 regulated open chromatin regions. (XLSX $14 \mathrm{~kb})$

\section{Abbreviations}

AP-1: Activated protein 1; CAD: Coronary artery disease; CDKN2B-

AS1: CDKN2BAS; eQTL: Expression quantitative trait locus; GWAS: Genomewide association studies; HAT: Histone acetyltransferase; HCASMC: Human coronary artery smooth muscle cells; HDAC: Histone deacetylase;

SMC: Smooth muscle cells; TF: Transcription factor

\section{Acknowledgements}

None.

\section{Funding}

T.Q. is supported by NIH grants R01HL109512, R01HL134817, R33HL120757, R01DK107437, R01 HL145708, and R01HL139478. C.L.M. is supported by ROOHL125912 (NIH).

\section{Availability of data and materials}

The datasets analyzed in the current study are available at Gene Ontology Omnibus (GEO): ATAC and H3K27ac ChIP sequencing data have been deposited to GEO with accession number GSE122758. (https://www.ncbi.nlm. nih.gov/geo/query/acc.cgi?acc=GSE122758).

JUN and TCF21 ChIP sequencing data: Sazonova O, et.al [23]. Characterization of TCF21 Downstream Target Regions Identifies a Transcriptional Network Linking Multiple Independent Coronary Artery Disease Loci. PLoS Genetics (PMID: 26020271) (https://doi.org/10.1371/journal.pgen.1005202) GSE61369. TCF21 RNA sequencing data: Nurnberg ST, et.al [22]. Coronary Artery Disease Associated Transcription Factor TCF21 Regulates Smooth Muscle Precursor Cells That Contribute to the Fibrous Cap. PLoS Genetics (PMID: 26020946) (https://doi.org/10.1371/journal.pgen.1005155) GSE44461.

\section{Authors' contributions}

QZ performed the experiments and analyzed the data. TN made the CRISPR lines and helped in the experiments. JBK performed RNAseq and analyzed the data. MP and RW helped in analyzing the data and contributed analyzing scripts. MN, JBK, and PC contributed reagents and materials. TQ, $\mathrm{QZ}$, and TN conceived and designed the experiments. RW, TN, JBK, and CLM discussed the manuscript. CLM critically reviewed the manuscript. TQ and QZ wrote the paper. All authors read and approved the final manuscript.

\section{Ethics approval and consent to participate}

HCASMC provided by commercial vendors Lonza, PromoCell, and Cell Applications were derived from deceased individuals. Because the donors were deceased, and only deidentified information was provided to the investigators; this work was not considered human research.

\section{Consent for publication}

Not applicable.

\section{Competing interests}

The authors declare that they have no competing interests.

\section{Publisher's Note}

Springer Nature remains neutral with regard to jurisdictional claims in published maps and institutional affiliations.

\section{Author details}

'Division of Cardiovascular Medicine and Cardiovascular Institute, School of Medicine, Stanford University, 300 Pasteur Dr., Falk CVRC, Stanford, CA 94305, USA. ${ }^{2}$ Center for Public Health Genomics, Department of Public Health Sciences, University of Virginia, Charlottesville, VA 22908, USA. ${ }^{3}$ Center for Public Health Genomics, Biochemistry and Molecular Genetics, University of Virginia, Charlottesville, VA, USA. ${ }^{4}$ Center for Public Health Genomics, Biomedical Engineering, University of Virginia, Charlottesville, VA, USA.

Received: 19 December 2018 Accepted: 3 April 2019

Published online: 23 April 2019

\section{References}

1. Howson JMM, et al. Fifteen new risk loci for coronary artery disease highlight arterial-wall-specific mechanisms. Nat Genet. 2017;49:1113-9.

2. Klarin D, et al. Genetic analysis in UK Biobank links insulin resistance and transendothelial migration pathways to coronary artery disease. Nat Genet. 2017:49:1392-7.

3. Nelson $\mathrm{CP}$, et al. Association analyses based on false discovery rate implicate new loci for coronary artery disease. Nat Genet. 2017:49:1385-91.

4. van der Harst P, Verweij N. The identification of 64 novel genetic loci provides an expanded view on the genetic architecture of coronary artery disease. Circ Res. 2017;122:433-43.

5. Maurano MT, et al. Systematic localization of common disease-associated variation in regulatory DNA. Science. 2012;337:1190-5.

6. Cui S, Ross A, Stallings N, Parker KL, Capel B, Quaggin SE. Disrupted gonadogenesis and male-to-female sex reversal in Pod1 knockout mice. Development. 2004;131:4095-105.

7. Cui S, Schwartz L, Quaggin SE. Pod1 is required in stromal cells for glomerulogenesis. Dev Dyn. 2003;226:512-22.

8. Hidai H, Bardales R, Goodwin R, Quertermous T, Quertermous EE. Cloning of capsulin, a basic helix-loop-helix factor expressed in progenitor cells of the pericardium and the coronary arteries. Mech Dev. 1998;73:33-43.

9. Lu J, Chang P, Richardson JA, Gan L, Weiler H, Olson EN. The basic helixloop-helix transcription factor capsulin controls spleen organogenesis. Proc Natl Acad Sci U S A. 2000;97:9525-30.

10. Lu J, Richardson JA, Olson EN. Capsulin: a novel bHLH transcription factor expressed in epicardial progenitors and mesenchyme of visceral organs. Mech Dev. 1998;73:23-32.

11. Lu JR, et al. Control of facial muscle development by MyoR and capsulin. Science. 2002;298:2378-81.

12. Quaggin $S E$, et al. The basic-helix-loop-helix protein pod 1 is critically important for kidney and lung organogenesis. Development. 1999;126:5771-83.

13. Robb L, et al. Epicardin: a novel basic helix-loop-helix transcription factor gene expressed in epicardium, branchial arch myoblasts, and mesenchyme of developing lung, gut, kidney, and gonads. Dev Dyn. 1998;213:105-13.

14. Schunkert $\mathrm{H}$, et al. Large-scale association analysis identifies 13 new susceptibility loci for coronary artery disease. Nat Genet. 2011;43:333-8.

15. Acharya A, et al. The bHLH transcription factor Tcf21 is required for lineage-specific EMT of cardiac fibroblast progenitors. Development. 2012:139:2139-49

16. Braitsch CM, Combs MD, Quaggin SE, Yutzey KE. Pod1/Tcf21 is regulated by retinoic acid signaling and inhibits differentiation of epicardium-derived cells into smooth muscle in the developing heart. Dev Biol. 2012;368:345-57. 
17. Lu X, et al. Genome-wide association study in Han Chinese identifies four new susceptibility loci for coronary artery disease. Nat Genet. 2012;44:890-4.

18. Liang J, et al. Single-trait and multi-trait genome-wide association analyses identify novel loci for blood pressure in African-ancestry populations. PLoS Genet. 2017;13:e1006728.

19. Liu B, et al. Genetic regulatory mechanisms of smooth muscle cells map to coronary artery disease risk loci. Am J Hum Genet. 2018;103:377-88.

20. Miller $\mathrm{CL}$, et al. Disease-related growth factor and embryonic signaling pathways modulate an enhancer of TCF21 expression at the $6 \mathrm{q} 23.2$ coronary heart disease locus. PLoS Genet. 2013;9:e1003652.

21. Miller $\mathrm{CL}$, et al. Coronary heart disease-associated variation in TCF21 disrupts a miR-224 binding site and miRNA-mediated regulation. PLoS Genet. 2014;10:e1004263.

22. Nurnberg ST, et al. Coronary artery disease associated transcription factor TCF21 regulates smooth muscle precursor cells that contribute to the fibrous cap. PLoS Genet. 2015;11:e1005155.

23. Sazonova $\mathrm{O}$, et al. Characterization of TCF21 downstream target regions identifies a transcriptional network linking multiple independent coronary artery disease loci. PLoS Genet. 2015;11:e1005202.

24. Karin M, Liu Z, Zandi E. AP-1 function and regulation. Curr Opin Cell Biol. 1997:9:240-6.

25. Chinenov Y, Kerppola TK. Close encounters of many kinds: Fos-Jun interactions that mediate transcription regulatory specificity. Oncogene. 2001;20:2438-52

26. Meng Q, Xia Y. c-Jun, at the crossroad of the signaling network. Protein Cell. 2011;2:889-98

27. Gosselin D, et al. Environment drives selection and function of enhancers controlling tissue-specific macrophage identities. Cell. 2014;159:1327-40.

28. Heinz S, et al. Effect of natural genetic variation on enhancer selection and function. Nature. 2013;503:487-92.

29. Vierbuchen T, et al. AP-1 transcription factors and the BAF complex mediate signal-dependent enhancer selection. Mol Cell. 2017;68:1067-1082 e1012.

30. Biddie SC, et al. Transcription factor AP1 potentiates chromatin accessibility and glucocorticoid receptor binding. Mol Cell. 2011;43:145-55.

31. Ng KW, Ridgway P, Cohen DR, Tremethick DJ. The binding of a Fos/Jun heterodimer can completely disrupt the structure of a nucleosome. EMBO J. 1997; 16:2072-85

32. Ye $Y$, Yang $X$, Long $B$, Pang $H$, Zhu $Y$, Zhang $S$. Association between a CCL17 genetic variant and risk of coronary artery disease in a Chinese Han population. Circ J. 2017:82:224-31

33. Miller $\mathrm{CL}$, et al. Integrative functional genomics identifies regulatory mechanisms at coronary artery disease loci. Nat Commun. 2016;7:12092108.

34. Farh KK, et al. Genetic and epigenetic fine mapping of causal autoimmune disease variants. Nature. 2015;518:337-43.

35. Turner AW, et al. Functional analysis of a novel genome-wide association study signal in SMAD3 that confers protection from coronary artery disease. Arterioscler Thromb Vasc Biol. 2016;36:972-83.

36. Hauberg ME, et al. Large-scale identification of common trait and disease variants affecting gene expression. Am J Hum Genet. 2017;100:885-94.

37. lyer $\mathrm{D}$, et al. Coronary artery disease genes SMAD3 and TCF21 promote opposing interactive genetic programs that regulate smooth muscle cell differentiation and disease risk. PLoS Genet. 2018;14:e1007681.

38. Cunnington MS, Santibanez Koref M, Mayosi BM, Burn J, Keavney B. Chromosome 9p21 SNPs associated with multiple disease phenotypes correlate with ANRIL expression. PLoS Genet. 2010;6:e1000899.

39. Jarinova O, et al. Functional analysis of the chromosome 9p21.3 coronary artery disease risk locus. Arterioscler Thromb Vasc Biol. 2009.

40. Holdt LM, et al. Alu elements in ANRIL non-coding RNA at chromosome 9 p21 modulate atherogenic cell functions through trans-regulation of gene networks. PLoS Genet. 2013:9:e1003588.

41. Visel A, et al. Targeted deletion of the 9p21 non-coding coronary artery disease risk interval in mice. Nature. 2010:464:409-12.

42. Lo Sardo $V$, et al. Unveiling the role of the most impactful cardiovascular risk locus through haplotype editing. Cell. 2018;175:1796-810 e1720.

43. Nikpay $M$, et al. A comprehensive 1,000 genomes-based genome-wide association meta-analysis of coronary artery disease. Nat Genet. 2015:47:1121-30.

44. Kim BJ, et al. TCF21 and the aryl-hydrocarbon receptor cooperate to activate a pro-atherosclerotic gene expression program. PLoS Genet. 2017;13:1006750.
45. Yu W, et al. Epigenetic silencing of tumour suppressor gene p15 by its antisense RNA. Nature. 2008:451:202-6.

46. Virmani R, Burke AP, Farb A, Kolodgie FD. Pathology of the vulnerable plaque. J Am Coll Cardiol. 2006:47:C13-8.

47. Pechlivanis $S$, et al. Risk loci for coronary artery calcification replicated at 9p21 and 6q24 in the Heinz Nixdorf Recall Study. BMC Med Genet. 2013;14:23.

48. Yang C, Shapiro LH, Rivera M, Kumar A, Brindle PK. A role for CREB binding protein and p300 transcriptional coactivators in Ets-1 transactivation functions. Mol Cell Biol. 1998;18:2218-29.

49. Qiu Y, Sharma A, Stein R. p300 mediates transcriptional stimulation by the basic helix-loop-helix activators of the insulin gene. Mol Cell Biol. 1998;18:2957-64.

50. Lee JS, See RH, Deng T, Shi Y. Adenovirus E1A downregulates cJun- and JunB-mediated transcription by targeting their coactivator p300. Mol Cell Biol. 1996;16:4312-26.

51. Tu WJ, et al. Priming of transcriptional memory responses via the chromatin accessibility landscape in T cells. Sci Rep. 2017;7:44825.

52. Hong CY, et al. Modulation of the expression and transactivation of androgen receptor by the basic helix-loop-helix transcription factor Pod-1 through recruitment of histone deacetylase 1. Mol Endocrinol. 2005:19:2245-57.

53. Tandon P, Miteva YV, Kuchenbrod LM, Cristea IM, Conlon FL. Tcf21 regulates the specification and maturation of proepicardial cells. Development. 2013;140:2409-21.

54. Consortium GT, et al. Genetic effects on gene expression across human tissues. Nature. 2017;550:204-13.

55. Nelson CP, et al. Genetically-determined height and coronary artery disease. N Engl J Med. 2015;372:1608-18.

56. Turner AW, et al. Functional interaction between COL4A1/COL4A2 and SMAD3 risk loci for coronary artery disease. Atherosclerosis. 2015;242:543-52.

57. Bengal $E$, et al. Functional antagonism between c-Jun and MyoD proteins: a direct physical association. Cell. 1992:68:507-19.

58. Romanoski CE, et al. Network for activation of human endothelial cells by oxidized phospholipids: a critical role of heme oxygenase 1. Circ Res. 2011;109:e27-41

59. Jiang $X$, Yang Z. Multiple biological functions of transcription factor 21 in the development of various cancers. Onco Targets Ther. 2018;11:3533-9.

60. Qin S, Li A, Yi M, Yu S, Zhang M, Wu K. Recent advances on antiangiogenesis receptor tyrosine kinase inhibitors in cancer therapy. $J$ Hematol Oncol. 2019;12:27.

61. Apte RS, Chen DS, Ferrara N. VEGF in signaling and disease: beyond discovery and development. Cell. 2019;176:1248-64.

Ready to submit your research? Choose BMC and benefit from:

- fast, convenient online submission

- thorough peer review by experienced researchers in your field

- rapid publication on acceptance

- support for research data, including large and complex data types

- gold Open Access which fosters wider collaboration and increased citations

- maximum visibility for your research: over $100 \mathrm{M}$ website views per year

At $\mathrm{BMC}$, research is always in progress.

Learn more biomedcentral.com/submission 